\title{
ANALYSE D'UNE EXPÉRIENCE MULTI-STATIONNELLE DE PROVENANCES D'ÉPICÉA (PICEA ABIES) KARST.)
}

\author{
J.-F, LACAZE \\ Station d'Amélioration des Arbres forestiers, \\ Centre national de Recherches forestières, 54 - Nancy \\ Institut national de la Recherche agronomique
}

\section{RESUME}

Cet article fait le bilan d'une expérimentation multi-stationnelle de provenances d'épicéa commencée en 1957.

L'analyse permet d'étudier le comportement de ces populations dans chaque station (3) et d'évaluer l'effet interaction et l'effet crise de transplantation.

Lanalyse critique de la méthodologie adoptée conduit à proposer une nouvelle méthode expérimentale d'étude de populations de conifêres, l'objectif étant de réduire les coûts et d'améliorer l'efficacité de la recherche,

\section{INTRODUCTION}

Le présent compte rendu a pour but de dresser un bilan de la première expérimentation française de conception moderne sur les provenances d'épicéa (Picea abies) qui a été engagéc au printemps 1957 (semis en pépinière). Les analyses présentées concernent les données recueillies entre cette date et le début de l'année 1969. c'est-à-dire pendant la période juvénile (12 ans après le semis, 8 ans après la plantation) couvrant \& la crise dite de transplantation *.

\section{I. - DESCRIPTION DE L'EXPERIENCE}

\section{1. - Provenances expérimentées}

Il s'agit de sources de graines françaises décrites sur le tableau 1 . On remarquera la présence d'une source artificielle \& Eclache\$, choisie dans le Massif Central, et d'une provenance autrichienne \& Lankowitz \& représentée dans une expérience antérieure. 
TABLEAU 1

Liste des provenances

\begin{tabular}{|c|c|c|c|}
\hline Provenance & Abréviation & $\begin{array}{l}\text { Latitude } \\
\text { (degrés) }\end{array}$ & $\begin{array}{l}\text { Altitude } \\
\text { (m) }\end{array}$ \\
\hline Forêt domaniale de Gérardmer (Vosges) . ......... & GERA I & $48^{\circ} 11^{*} \mathrm{~N}$ & 800 \\
\hline Peuplement \& plus \& du Kertoff & & & \\
\hline Forèt domaniale de Gérardmer (Vosges) ............. & GERA 11 & $48^{\circ} 04^{*} \mathrm{~N}$ & 900 \\
\hline Forêt domaniale de Bonnétage (Doubs) ............... & BONE I & $47^{\circ} 11^{\prime} \mathrm{N}$ & 880 \\
\hline Forêt communale des Rousses (Jura - Massif de Risoux) & ROUS & $46^{\circ} 31^{*} \mathrm{~N}$ & 1.250 \\
\hline Forêt communale de la Grand'Côte (Doubs) ............. & GR-CO I & $46^{\circ} 48^{\prime} \mathrm{N}$ & 950 \\
\hline Forèt communale d'Esserval-Tartre (Jura) ............. & ES-TA II & $46^{\circ} 50^{\circ} \mathrm{N}$ & 820 \\
\hline Forèt communale de Saint-Laurent (Jura) ........... & SLAU I & $46^{\circ} 33^{\circ} \mathrm{N}$ & 980 \\
\hline Forêt communale de Chamrousse (Isère-Oisans) ........ & CAMR & $45^{\circ} 06^{\circ} \mathrm{N}$ & 1.100 \\
\hline Forêt communale de Verdaches (Basses-Alpes) ......... & VERD & $44^{*} 16^{*} \mathrm{~N}$ & 1.350 \\
\hline Forét domaniale de la Grande-Chartreuse (Isère) ,..... & GR-CH & $45^{\circ} 22 \cdot \mathrm{N}$ & 800 \\
\hline Forêt communale d'Autrans (Isère-Vercors) ............ & AUTR & $45^{*} 10^{*} \mathrm{~N}$ & 1.250 \\
\hline Forêt communale de Peisey-Nancroix (Savoie Tarentaise) & PE-NA I & $45^{*} 32 * \mathrm{~N}$ & 1.400 \\
\hline $\begin{array}{c}\text { Forêt communale de Saint-Etienne-en-Devoluy (Hautes- } \\
\text { Alpes) } \ldots \ldots \ldots \ldots \ldots \ldots \ldots \ldots \ldots \ldots \ldots \ldots \ldots \ldots \ldots \ldots \ldots \ldots\end{array}$ & SE-DE & $44^{\circ} 42^{\prime} \mathrm{N}$ & 1.500 \\
\hline Forét domaniale de IEclache (Puy-de-Dôme) ........... & ECLA & $45^{\circ} 45^{\circ} \mathrm{N}$ & 950 \\
\hline 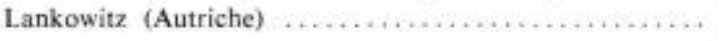 & LANK II & $47^{\circ} 05^{\circ} \mathrm{N}$ & 1.000 \\
\hline
\end{tabular}


TABLEAU 2

Description des stations

\begin{tabular}{|c|c|c|c|c|c|c|c|}
\hline Localisation & Latitude & Longitude & $\begin{array}{c}\text { Région } \\
\text { géographique }\end{array}$ & $\begin{array}{l}\text { Type de } \\
\text { station }\end{array}$ & $\begin{array}{l}\text { Pluvio- } \\
\text { métrie } \\
\text { (mm) }\end{array}$ & $\begin{array}{c}\text { Alti- } \\
\text { tude } \\
\text { (m) }\end{array}$ & Observations \\
\hline Sylvetum de Grandsagnes ....... & $43^{\circ} 30^{\prime} \mathrm{N}$ & $2^{\circ} 44^{\prime} \mathrm{E}$ & $\begin{array}{l}\text { Monts du Somail. } \\
\text { Relief sud du } \\
\text { Massif Central }\end{array}$ & $\begin{array}{l}\text { Lande à callune } \\
\text { avec Genista pilo- } \\
\text { sa. }\end{array}$ & 1.430 & 900 & Effet de crête. \\
\hline Forèt communale d'Epinal ..... & $48^{\circ} 05^{\circ} \mathrm{N}$ & $6^{\circ} 34^{\prime} \mathrm{E}$ & Basses Vosges & $\begin{array}{l}\text { Hétraie à luzule } \\
\text { blanchâtre. }\end{array}$ & 1.000 & 390 & $\begin{array}{l}\text { Plantation après coupe } \\
\text { rase de la hétraie. }\end{array}$ \\
\hline Forêt domaniale du Guéry .... & $45^{\circ} 37^{\prime} \mathrm{N}$ & $2^{\circ} 50^{\prime} \mathrm{E}$ & Mont-Dore & Pelouse d'altitude. & 1.600 & 1.350 & $\begin{array}{l}\text { Limite de la végétation } \\
\text { forestière en altitude. }\end{array}$ \\
\hline
\end{tabular}




\section{2. - Stade pépinière (Amance, près de Nancy)}

- semis en plein, selon un dispositif en blocs incomplets, au printemps 1957,

- repiquage à deux ans, selon un dispositif en blocs complets, au printemps 1959 .

\section{3. - Stade plantations comparatives}

Les plants ont été répartis sur trois stations sommairement décrites sur le tableau 2.

Il s'agit de trois stations dont les caractéristiques écologiques sont très différentes et qui représentent trois zones d'introduction classiques de l'épicéa.

Les dispositifs sont présentés sur le tableau 3, la densité de plantation correspondant à l'écartement $2 \times 2 \mathrm{~m}$ dans les trois stations.

TABLEAU 3

Dispositifs des plantations comparatives

\begin{tabular}{|c|c|c|c|c|c|c|}
\hline \multirow{2}{*}{ Plantations } & \multicolumn{4}{|c|}{ Caractéristiques des dispositifs } & \multirow{2}{*}{$\begin{array}{c}\text { Nombre de } \\
\text { plants par } \\
\text { parcelle } \\
\text { unitaire }\end{array}$} & \multirow{2}{*}{$\begin{array}{c}\text { Date } \\
\text { de } \\
\text { plantation }\end{array}$} \\
\hline & $\begin{array}{l}\text { Nombre de } \\
\text { provenances }\end{array}$ & $\begin{array}{l}\text { Nombre de } \\
\text { répétitions }\end{array}$ & $\begin{array}{c}\text { Nombre de } \\
\text { provenances } \\
\text { par bloc }\end{array}$ & $\begin{array}{c}\text { Nombre de } \\
\text { blocs }\end{array}$ & & \\
\hline Grandsagnes & 7 & 4 & 4 & 7 & 72 & oct. 1960 \\
\hline Epinal...$\ldots$. & 13 & 4 & 4 & 13 & 72 & mars 1961 \\
\hline Guéry $\ldots \ldots$. & 13 & 4 & 4 & 13 & 72 & avril 1961 \\
\hline \multicolumn{3}{|c|}{ Provenances } & Grandsagnes & Epinal & & Guéry \\
\hline \multirow{6}{*}{\multicolumn{3}{|c|}{ 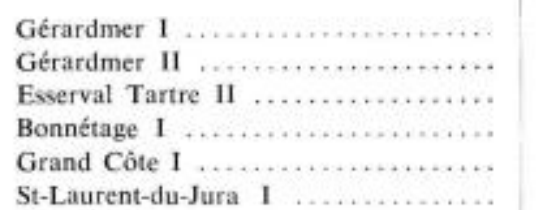 }} & $\mathrm{x}$ & $\mathrm{x}$ & & $\mathrm{x}$ \\
\hline & & & & $\mathrm{x}$ & & \\
\hline & & & $\mathrm{x}$ & $\mathrm{x}$ & & $\mathrm{x}$ \\
\hline & & & & $\mathrm{x}$ & & $\mathrm{x}$ \\
\hline & & & $\mathrm{x}$ & $x$ & & $\mathrm{x}$ \\
\hline & & & & $\mathrm{x}$ & & \\
\hline \multicolumn{3}{|c|}{ Les Rousses $\ldots \ldots \ldots \ldots \ldots \ldots \ldots \ldots$} & & & & $\mathrm{x}$ \\
\hline \multicolumn{3}{|c|}{ Grande Chartreuse............} & $\mathrm{x}$ & $\mathrm{x}$ & & $\mathrm{x}$ \\
\hline \multirow{2}{*}{\multicolumn{3}{|c|}{$\begin{array}{l}\text { Chamrousse } \ldots \ldots \ldots \ldots \\
\text { Autrans } \ldots \ldots \ldots \ldots \ldots\end{array}$}} & & $\mathrm{x}$ & & $\mathrm{x}$ \\
\hline & & & & $\mathrm{x}$ & & $\mathrm{x}$ \\
\hline \multirow{2}{*}{\multicolumn{3}{|c|}{$\begin{array}{l}\text { Peisey-Nancroix } 1 \text {........................ } \\
\text { St-Ftienne-en-Dévoluy }\end{array}$}} & $x$ & & & $\mathrm{x}$ \\
\hline & & & & $\mathbf{x}$ & & $\mathrm{x}$ \\
\hline \multirow{2}{*}{\multicolumn{3}{|c|}{$\begin{array}{l}\text { Verdaches } \ldots \ldots \ldots \ldots \ldots \ldots \ldots \ldots \ldots \ldots \ldots \ldots \ldots \ldots \ldots \ldots \ldots \ldots \ldots \ldots \ldots \ldots \\
\text { Eclache }\end{array}$}} & $\mathrm{x}$ & $\mathrm{x}$ & & $\mathrm{x}$ \\
\hline & & & & $\mathrm{x}$ & & $\mathrm{x}$ \\
\hline \multicolumn{3}{|c|}{ Lankowitz II ....................... } & $\mathrm{x}$ & $\mathrm{x}$ & & $\mathrm{x}$ \\
\hline
\end{tabular}

\section{4. - Mesures effectuées}

Mesures de vigueur (âges à partir de la graine).

\begin{tabular}{|c|c|c|c|c|c|c|c|c|}
\hline Hauteur & Hauteur & Hauteur & Flèche & Flèche & Flèche & Flèche & Flèche & Flèche \\
\hline à 2 ans & à 4 ans & a 6 ans & $7^{*}$ année & 8 année & $9^{*}$ année & $10^{\circ}$ année & $11^{*}$ annće & $12^{\prime}$ année \\
\hline P 1959 (1) & P 1961 & P 1963 & A 1963 & A 1964 & A 1965 & A 1966 & A 1967 & A 1968 \\
\hline
\end{tabular}

(1) $\mathbf{P}=$ printemps, $\mathbf{A}=$ automne, 
Mesures de caractères phénologiques: débourrement végétatif.

Pépinière (Amance) : P 1960 (début de la $4^{*}$ année).

Grandsagnes: P 1963 (début de la $7^{*}$ année).

Epinal: P 1962 [pousse terminale, pousses latérales] (début de la $6^{\circ}$ année):

P 1963 (đébut de la $7^{*}$ année):

P 1964 (début de la $8^{*}$ année).

Guéry: P 1963 (début de la $7^{*}$ année);

P 1964 (début de la $8^{\circ}$ année).

Fréquence de $2^{*}$ pousse.

Pépinière (Amance) : été $1960\left(4^{*}\right.$ année).

Mesures diverses: dégâts de gelée.

Epinal: 1962 [pousse terminale, pousses latérales] ( $6^{\circ}$ année).

Code de notation du débourrement.

0 - Bourgeon dormant;

1 - Bourgeon gonflé :

2 - Extrémité des aiguilles apparentes ou capuchon du bourgeon soulevé ;

3 - Pousse d'une longueur inférieure à $2 \mathrm{~cm}$;

4 - Pousse d'une longueur comprise entre 2 et $4 \mathrm{~cm}$;

5 - Pousse d'une longueur supérieure à $4 \mathrm{~cm}$.

Code de notation des 2 " pousses.

0 - pas de $2^{\circ}$ pousse :

$1-2^{*}$ pousse sur pousses latérales;

$2-2^{*}$ pousse sur pousses latérales et terminale.

Code de notation des dégâts de gelée.

Pousse terminale:

1 - dégât :

2 - pas de dégât.

Pousses latérales:

2 - plus de $80 \%$ des pousses gelées:

1 - moins de $80 \%$ des pousses gelées:

0 - pas de dégât.

\section{II. - RESULtATS - ClASSEMENTS DES PROVENANCES PAR STATION}

Le bilan à la fin du stade pépinière a été analysé par Bouvarel (1). Nous considérons ici les résultats obtenus au cours de la période de huit ans suivant la plantation sur le terrain.

\section{1. - Grandsagnes}

Les figures 1 et 2 (pousses et hauteurs totales annuelles) montrent qu'il n'existe aucune différence significative entre provenances pendant toute la période considé-

(1) Linfluence de l'origine des graines d'épicéa sur la croissance en pépinière, la précocité et la fréquence des pousses d'aoút. Annales de FEcole Nationale dés Eaux ê Foréts, 1962, Tome 19 (3). 


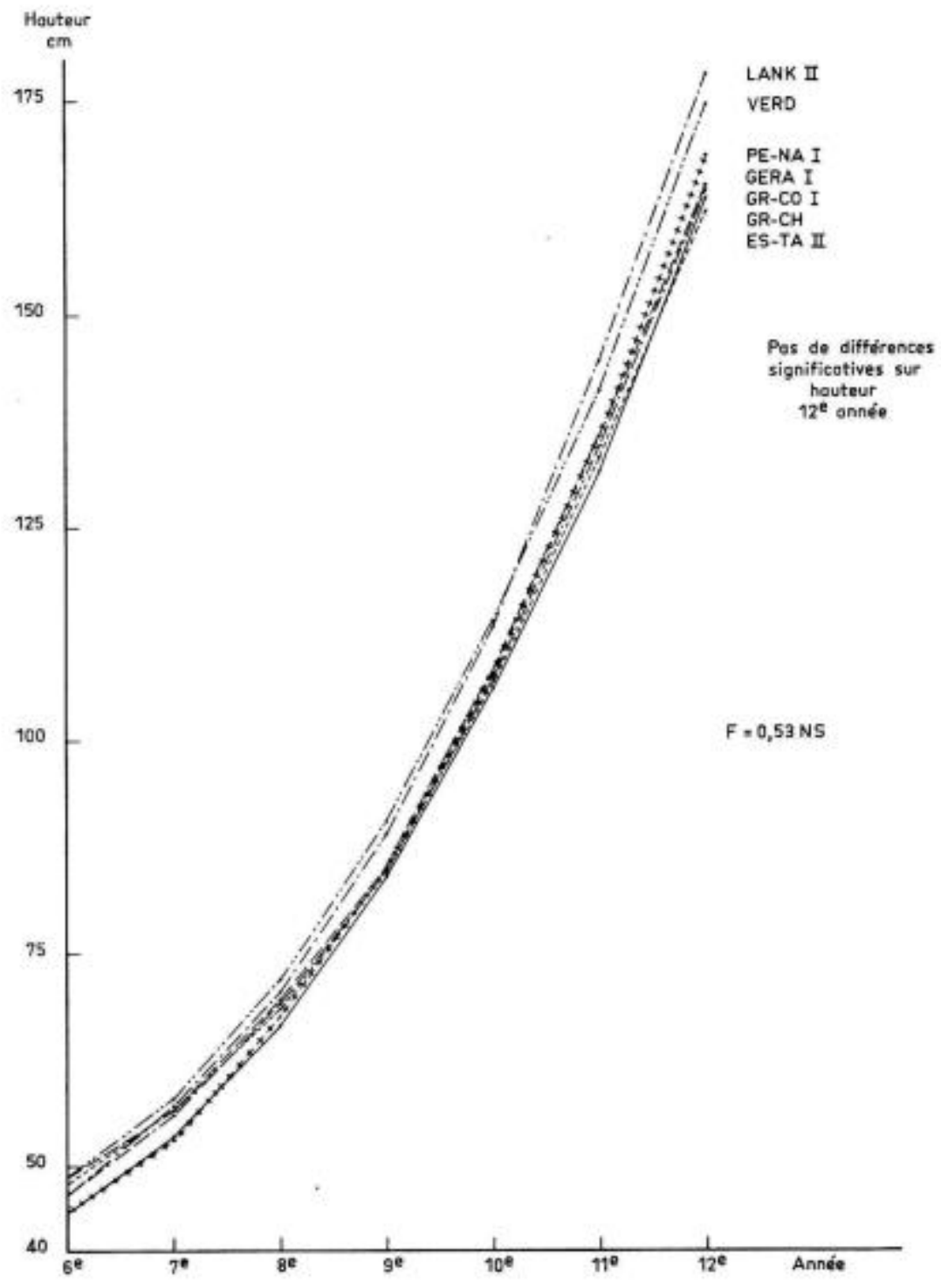

Fig. 1, - Grandsagnes: ćvolution des hauteurs totales moyennes des diverses provenances 


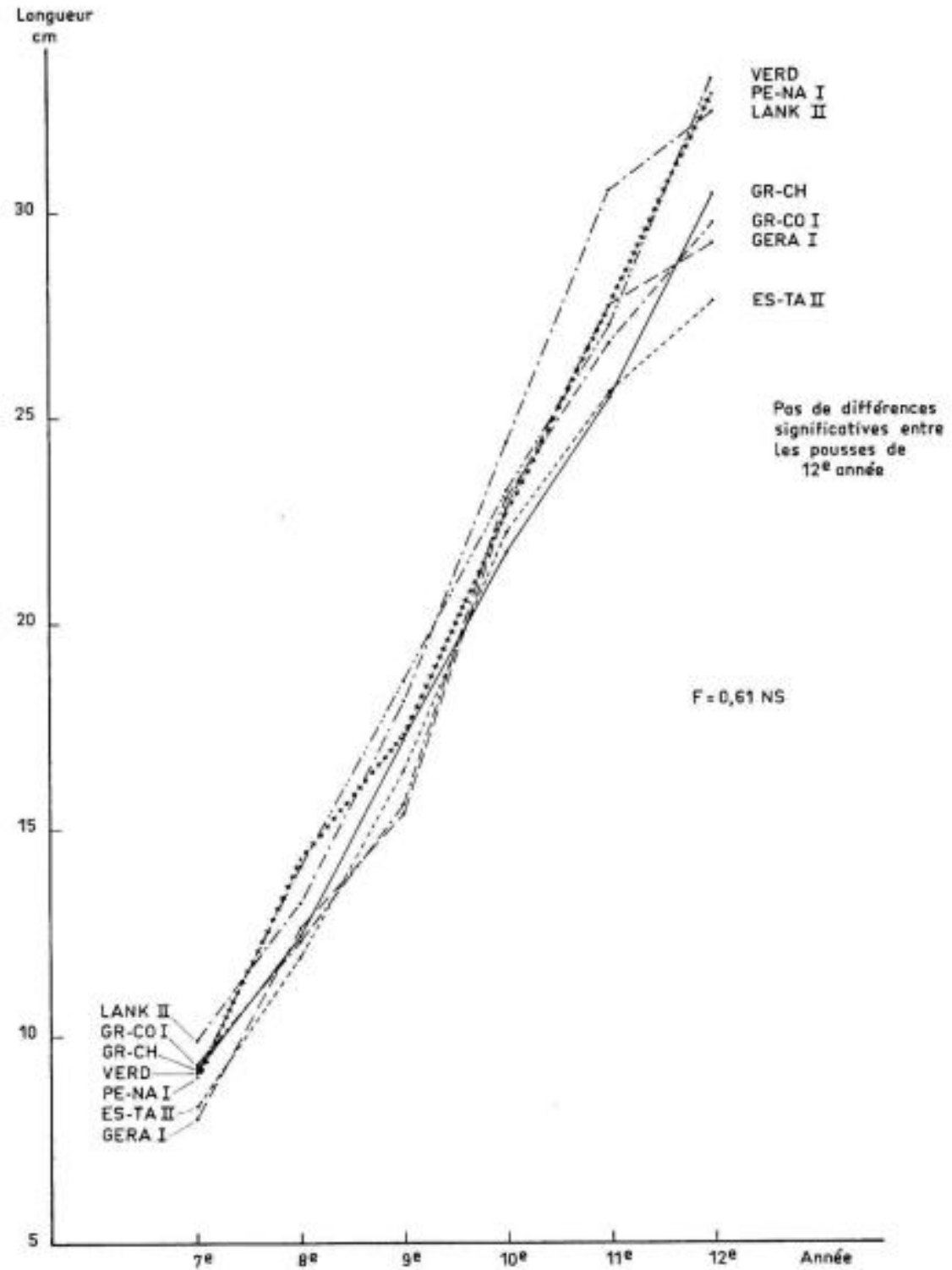

Fic. 2. - Grandsagnes: évolution des pousses annuelles moyennes des diverses provenances 
rée (1). Nous remarquerons seulement que sur les deux provenances vigoureuses en pépinière, Lankowitz et Gérardmer, la première se maintient en tête et la deuxième apparaît en fin de liste pour le caractère hauteur totale à 12 ans.

Le classement de débourrement ci-dessous (moyenne des notes de débourrement):

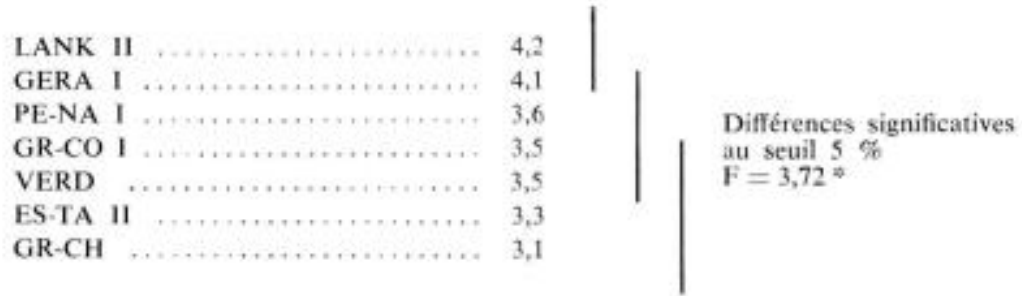

confirme la précocité des provenances Lankowitz et Gérardmer et la tardiveté d'Esserval-Tartre et de Grande-Chartreuse.

\section{2. - Epinal}

\subsection{Mesures de vigueur.}

Les hauteurs et pousses annuelles se trouvent sur les figures 3 et 4 . En ce qui concerne les hauteurs totales, on enregistre, huit ans après plantation, un classement très voisin de celui obtenu en pépinière. Un lot de tête groupe les provenances Eclache et Géradmer, suivi de près par Lankowitz, Bonnétage, Chamrousse et Esserval-Tartre; un troisième groupe rassemble les autres provenances de la partie supérieure du deuxième plateau du Jura, Grand-Côte et Saint-Laurent, ainsi que les sources alpines Verdaches et Autrans; enfin, Saint-Etienne-en-Dévoluy se détache en queue de classement.

En admettant qu'une plantation d'épicéa ne nécessite plus d'interventions importantes sous forme de dégagement lorsque les plants atteignent en moyenne $1 \mathrm{~m}$ de haut, on constate que dans une station de basse altitude comme Epinal. l'utilisation de provenances à croissance initiale rapide (Eclache ou Gérardmer) permet de réaliser une économie d'un ou deux dégagements selon que l'on se réfère à une provenance moyenne ou à une provenance peu vigoureuse (Saint-Etienne-en-Dévoluy). Dans le cas présent, les provenances vigoureuses ont été malheureusement vulnérables aux gelées tardives (parce que précoces, voir ci-dessous), ce qui a contrarié leur croissance initiaie et réduit d'autant l'avantage signalé pour les dégagements.

L'examen de la figure montrant l'évolution des flèches terminales suggère les commentaires suivants :

- Pour une majorité de provenances, il semble que la longueur des flèches annuelles tend à se stabiliser en 1968 (12" année) $(50 \mathrm{~cm}$ environ pour les meilleures provenances). Certaines au contraire produisent encore, en 1968, des

(1) Dans les analyses de variances concernant les pousses annuelles, les calculs ont été effectués à partir de la variable Logarithme népérien de la longueur (transformation normalisante). 


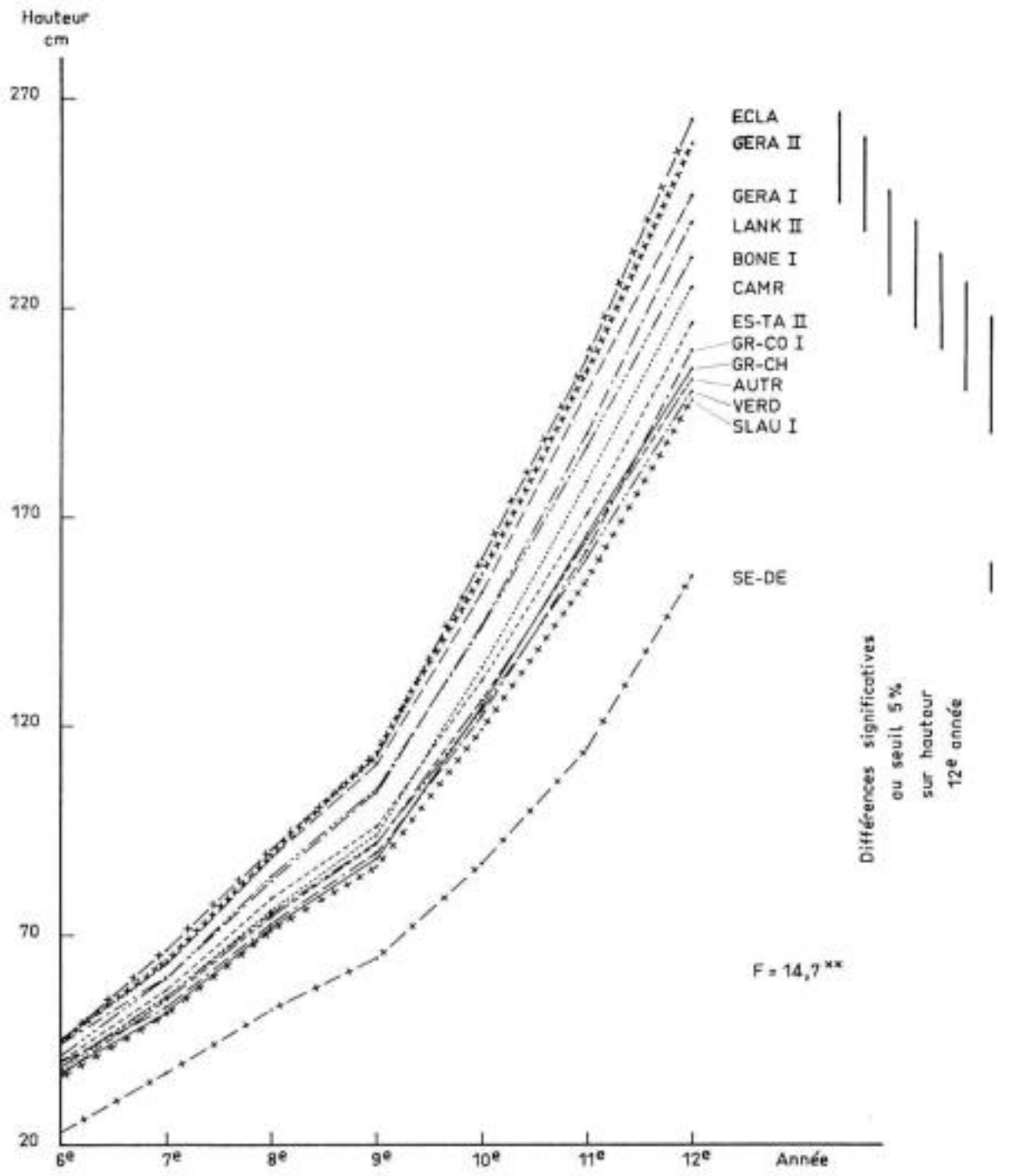

FIG. 3. - Epinal : évolution des hauteurs totales moyennes des diverses provenances 


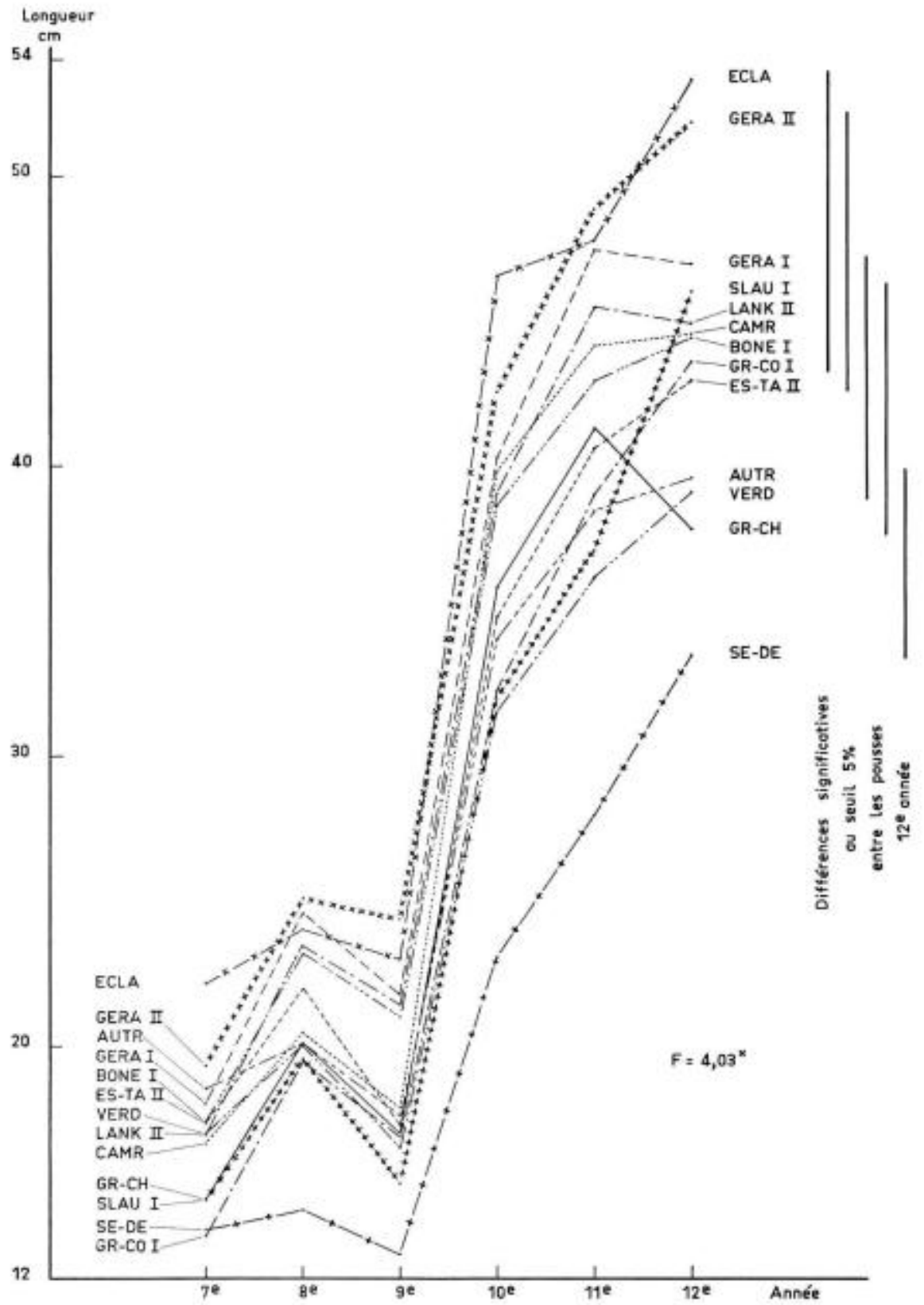

Fic. 4. - Epunal: évolution des pousses anmuelles moyennes des diverses provenances 
pousses terminales annuelles en forte progression sur l'année précédente; il s'agit en particulier de Saint-Etienne-en-Dévoluy, Grand-Côte et Saint-Laurent, ces deux dernières correspondant à la même région de provenances. Il s'agirait donc de populations à croissance initiale lente, mais dont \&l'accélération * de la vitesse d'élongation s'étalerait sur un plus grand nombre d'années. Les provenances Eclache et Gérardmer II cumulent les deux avantages, croissance initiale rapide et progression constante de la vigueur des flèches terminales.

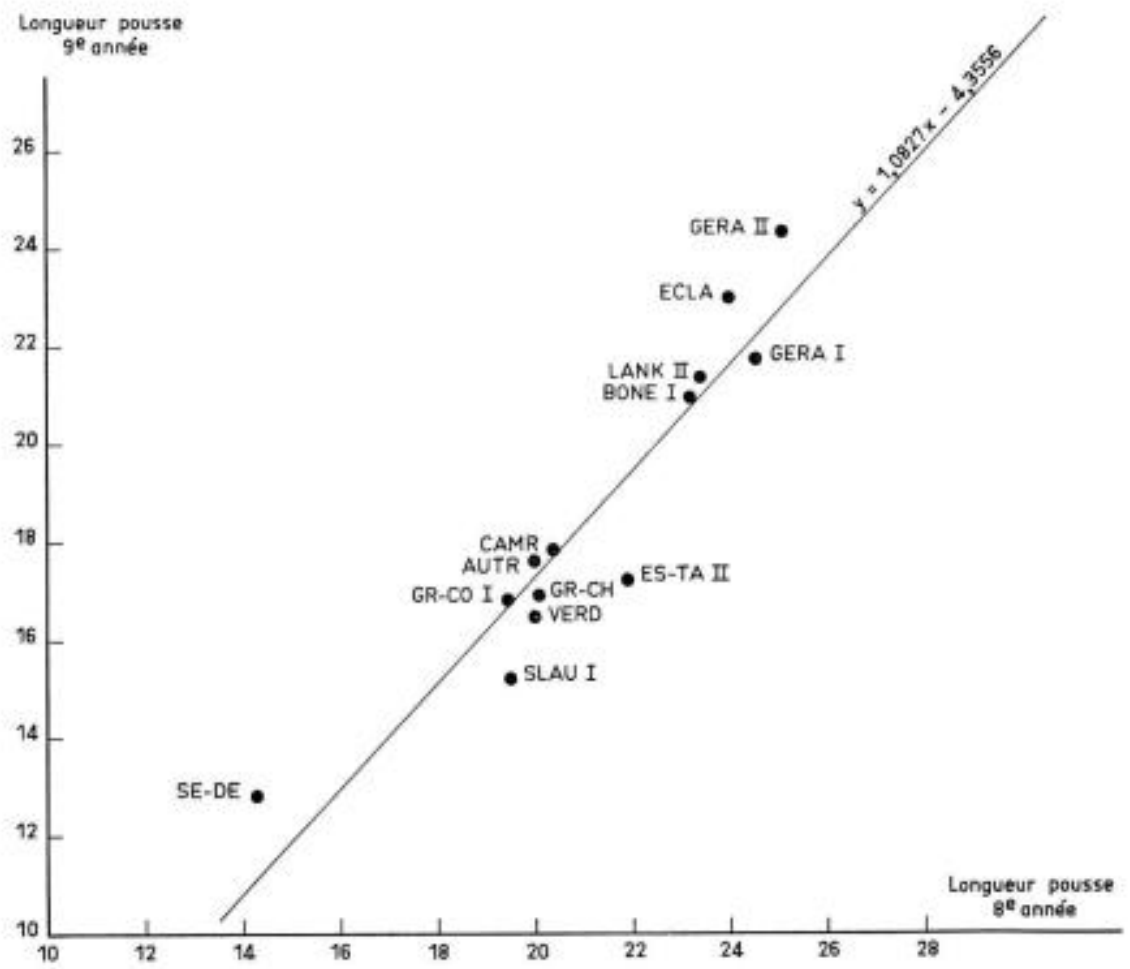

F16. 5, - Epinal: régression entre la pousse de la $8^{\circ}$ année et de la $9^{\circ}$ année (sécheresse exceptionnelle en 1964 )

- Les flèches de l'année $1965 \quad\left(9^{\circ}\right.$ année $)$ sont inférieures à celles des années précédentes, quelle que soit la provenance. $\mathrm{Ce}$ phénomène s'expliqué en examinant les chutes d'eau des années en cause.

\begin{tabular}{|c|c|c|c|c|c|c|c|}
\hline Années & 1961 & 1962 & 1963 & 1964 & 1965 & 1966 & 1967 \\
\hline $\begin{array}{l}\text { Chutes d'eau to- } \\
\text { tales }(\mathrm{mm})\end{array}$ & 1.098 & 966 & 981 & 763 & 1.390 & 1.242 & 1.194 \\
\hline $\begin{array}{l}\text { Chutes d'eau de } \\
\text { mai à septembre. }\end{array}$ & 420 & 394 & 541 & 297 & 676 & 454 & 546 \\
\hline
\end{tabular}


On enregistre, en 1964, un déficit de pluviosité de l'ordre de $25 \%$ qui se traduit par une réduction de l'élongation l'année suivante, en 1965. Chez l'épicéa, la capacité d'élongation de l'année $n$ serait inscrite dans le bourgeon terminal formé à la fin de l'année $n-1$, et la masse de ce bourgeon dépend probablement des conditions météorologiques de l'année $n-1$.

La question se pose de savoir si les diverses provenances ont accusé un déficit d'élongation identique en 1965. La régression entre pousse 1964 et pousse 1965 (voir figure 5) indique une très forte liaison entre ces deux mesures $\left(r=0,94^{* * *}\right.$ ). Globalement, les diverses populations étudiées auraient donc un comportement assez peu différent au cours d'une saison particulièrement sèche. On remarque, néanmoins, que pour les deux provenances très vigoureuses, Gérardmer II et Eclache, et la provenance méridionale, Saint-Etienne-en-Dévoluy, le déficit semble moins marqué. Les deux premières ont peut-être mis en jeu des réserves compensant des conditions de nutrition médiocre de l'année sèche. La troisième pourrait bénéficier d'une aptitude particulière à se développer sous climat sec. Au contraire, Esserval-Tartre et SaintLaurent (Jura) semblent plus particulièrement pénalisées; ces populations se développent naturellement sous un climat très arrosé.

\subsection{Débourrement végétatif.}

Les diverses notations de débourrement aboutissent à un classement pour la tardiveté voisin de celui établi au stade pépinière. Nous avons présenté ci-dessous, les seuls résultats de la mesure du printemps 1964 (début de la $8^{*}$ année).

\begin{tabular}{|c|c|c|}
\hline Provenances & $\begin{array}{c}\text { Proportion de plants } \\
\text { non débourrés (tardifs) } \\
\text { le } 12-5-64\end{array}$ & \\
\hline ECLA $\ldots \ldots \ldots \ldots$ & 59,4 & \\
\hline GERA II ............. & 64,3 & \\
\hline GERA I $\ldots \ldots \ldots$ & 68,0 & \\
\hline LANK II ............ & 74,8 & \\
\hline SLAU $1 \ldots \ldots \ldots$ & 77,8 & 1 \\
\hline SE-DE $\ldots \ldots \ldots \ldots$ & 81,1 & 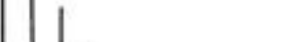 \\
\hline BONE I .............. & 91,3 & 1 \\
\hline ES-TA $11 \ldots \ldots \ldots$ & 93,9 & \\
\hline GR-CO I ............. & 94,3 & \\
\hline VERD $\ldots \ldots \ldots \ldots$ & 94,7 & | \\
\hline GR-CH $\ldots \ldots \ldots$ & 97,6 & \\
\hline CAMR ............. & 99,4 & $\mathrm{~F}=8,19 * *$ \\
\hline AUTR $\ldots \ldots \ldots \ldots$ & 99,4 & \\
\hline & & $\begin{array}{c}\text { Différences significatives } \\
\text { au seuil } 5 \%\end{array}$ \\
\hline
\end{tabular}

On retrouve, dans le groupe précoce, les populations à croissance rapide : Gérardmer, Eclache et Lankowitz, et parmi les tardives: les populations des Alpes (sauf Saint-Etienne-en-Dévoluy) et Bonnétage. 


\subsection{Dégâts dus aux gelêes tardives.}

Les dégâts dus à la première gelée tardive, celle de 1962, ont été plus marqués sur les provenances précoces, ce qui démontre l'intérêt de sélectionner des populations tardives pour les stations très exposées aux gelées de printemps.

\begin{tabular}{|c|c|}
\hline Provenances & $\begin{array}{l}\text { Proportion de plants ayant } \\
\text { la pousse terminale gelée en } 1962\end{array}$ \\
\hline GERA I,$\ldots \ldots \ldots \ldots \ldots \ldots \ldots$ & 15,7 \\
\hline LANK $\| \ldots \ldots \ldots \ldots \ldots \ldots$ & 14,5 \\
\hline 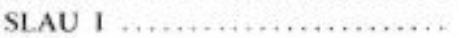 & 13,2 \\
\hline ECIAA,$\ldots \ldots \ldots \ldots \ldots$ & 10,2 \\
\hline GERA II $\ldots \ldots \ldots \ldots \ldots \ldots \ldots$ & 9,1 \\
\hline SE-DE,$\ldots \ldots \ldots \ldots \ldots \ldots \ldots$ & 8,5 \\
\hline ES-TA $I 1, \ldots \ldots \ldots \ldots \ldots \ldots \ldots$ & 7,3 \\
\hline $\mathrm{GR}-\mathrm{CH} \quad \ldots \ldots \ldots \ldots \ldots \ldots \ldots \ldots$ & 7.2 \\
\hline GR.CO I , , . . . . . . . . . . & 5,6 \\
\hline CAMR $\ldots \ldots \ldots \ldots \ldots \ldots \ldots$ & 4,5 \\
\hline AUTR $\ldots \ldots \ldots \ldots \ldots \ldots \ldots$ & 4,3 \\
\hline VERD $\ldots \ldots \ldots \ldots \ldots \ldots \ldots \ldots$ & 2,0 \\
\hline BONE $1 \ldots \ldots \ldots \ldots \ldots \ldots$ & 1,4 \\
\hline
\end{tabular}

Coefficients de corrélation entre les notations de débourrement et de dégâts de gelée

Débourrement 1962, pousse terminale . DÉbourrement 1962, pousses latérales Débourrement 1963, pousse terminale. Débourrement 1964, pousse terminale

\begin{tabular}{|c|c}
$\begin{array}{c}\text { Pourcentage de plants } \\
\text { ayant la pousse } \\
\text { terminale gelée en } 1962\end{array}$ & $\begin{array}{c}\text { Notation de dégâts } \\
\text { sur pousses latérales }\end{array}$ \\
\hline $0,68^{\text {ee }}$ & \\
$0,67^{\text {te }}$ & $0,66^{*}$ \\
$0,71^{\text {*e }}$ & \\
$0,79^{\text {*a* }}$ &
\end{tabular}

II est également intéressant de savoir s'il existe une relation du même genre au niveau individuel, c'est-à-dire de vérifier si, dans chaque provenance, les plants ayant subi des dégâts de gelée sont également les plus précoces.

Le tableau ci-dessous donne, pour chaque population, les valeurs moyennes des notes de débourrement du printemps 1962 de la sous-population de plants endommagés (bourgeon terminal) et celles de la sous-population de plants indemnes, à la suite de la gelée tardive du même printemps 1962.

Les sous-populations de plants gelés sont toujours plus précoces que celles de plants indemnes (bien que les différences ne soient pas toujours significatives), ce qui justifie de retenir le caractère débourrement végétatif en tant que critère de sélection individuelle, pour l'obtention de variétés à utiliser sur station exposée aux gelées tardives. 
Les plants gelés sont, en effet, pénalisés dans leur croissance initiale, alors que celle-ci revêt une grosse importance pratique (nombre de dégagements).

\begin{tabular}{|c|c|c|c|}
\hline Provenances & $\begin{array}{l}\text { Débourrement } \\
\text { Sous-population } \\
\text { de plants gelés }\end{array}$ & $\begin{array}{l}\text { Débourrement } \\
\text { Sous-population } \\
\text { de plants indemnes }\end{array}$ & Test $\mathrm{t}$ \\
\hline 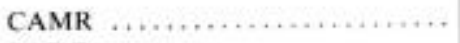 & 2.8 & 2,7 & $0,20 \mathrm{NS}$ \\
\hline SE-DE $\ldots \ldots \ldots \ldots \ldots \ldots \ldots \ldots$ & 3.7 & 3,6 & $0,37 \mathrm{NS}$ \\
\hline SLAU $1, \ldots \ldots \ldots \ldots \ldots \ldots \ldots$ & 3,2 & 3.2 & - NS \\
\hline GERA II $\ldots \ldots \ldots \ldots \ldots \ldots \ldots$ & 3,7 & 3,3 & $1,2 \mathrm{NS}$ \\
\hline ES.TA $\| \quad \ldots \ldots \ldots \ldots \ldots \ldots$. & 3,4 & 2,8 & $2,3^{*}$ \\
\hline AUTR $\ldots \ldots \ldots \ldots \ldots \ldots \ldots \ldots$ & 3.7 & 2,6 & $2,9^{\circ}$ \\
\hline ECLA $\ldots \ldots \ldots \ldots \ldots \ldots \ldots$ & 3,6 & 3,5 & $0,3 \mathrm{NS}$ \\
\hline VERD $\ldots \ldots \ldots \ldots \ldots \ldots \ldots \ldots$ & 3,5 & 2,9 & $1,1 \mathrm{NS}$ \\
\hline LANK $11 \ldots \ldots \ldots \ldots \ldots \ldots \ldots$ & 3,4 & 3,0 & $2,07^{*}$ \\
\hline GR-CO I $\ldots \ldots \ldots \ldots \ldots \ldots$ & 3,0 & 2,7 & $2,5^{\circ}$ \\
\hline GERA $1, \ldots, \ldots, \ldots \ldots \ldots \ldots$ & 4,1 & 3,5 & $2,3^{\circ}$ \\
\hline GR-CH $\ldots \ldots \ldots \ldots \ldots \ldots \ldots$ & 3,0 & 2,7 & $3,1^{*}$ \\
\hline
\end{tabular}

$\mathrm{Si}$ nous considérons les coefficients de corrélation intrapopulation des mesures de vigueur (hauteur totale à 6 ans et 12 ans) d'une part et la notation de dégâts de gelée du printemps de la sixième année d'autre part, nous constatons qu’en gros, cette pénalisation serait d'autant plus marquée et son effet prolongé que la provenance est plus précoce.

\begin{tabular}{|c|c|c|}
\hline Provenances & Hauteur à 6 ans/gelée & Hauteur à 12 ans/gelée \\
\hline GERA $11, \ldots \ldots \ldots \ldots \ldots$ & $-0,270^{\circ 66}$ & $-0,427^{\circ+6}$ \\
\hline GERA I $\ldots \ldots \ldots \ldots \ldots \ldots$ & $-0,337^{=* *}$ & $-0,324^{46 *}$ \\
\hline ECLA,$\ldots \ldots \ldots \ldots \ldots \ldots$ & $-0,337^{* 1 * a}$ & $-0.15^{4}$ (limite) \\
\hline LANK $\| \ldots \ldots \ldots \ldots$ & $-0,218=$ & $-0,04 \mathrm{NS}$ \\
\hline ES-TA $\| \ldots \ldots \ldots \ldots \ldots$ & $-0,279 * \%$ & $-0,171=$ \\
\hline VERD $\ldots \ldots \ldots \ldots \ldots \ldots \ldots \ldots \ldots \ldots$ & $-0,075 \mathrm{NS}$ & $-0,071 \mathrm{NS}$ \\
\hline CAMR $\ldots \ldots \ldots \ldots \ldots \ldots$ & $-0,277^{\circ 6}$ & $-0,201^{*}$ \\
\hline GR-CH $\ldots \ldots \ldots \ldots \ldots \ldots \ldots$ & $-0,245^{\circ 6}$ & $-0,005 \mathrm{NS}$ \\
\hline Ensemble des populations .... & $-0,247^{040}$ & $-0,129^{\circ}$ \\
\hline
\end{tabular}

Ces diverses relations entre précocité et dégâts de gelée, au niveau individuel et au niveau de la moyenne de populations, semblent, a priori, évidentes. Il ne nous a pas paru inutile d'en apporter des preuves chiffrées.

$$
\text { 2.3. - Guéry }
$$

Les mesures de vigueur sont présentées, comme précédemment, sur les figures 6 et 7 . 


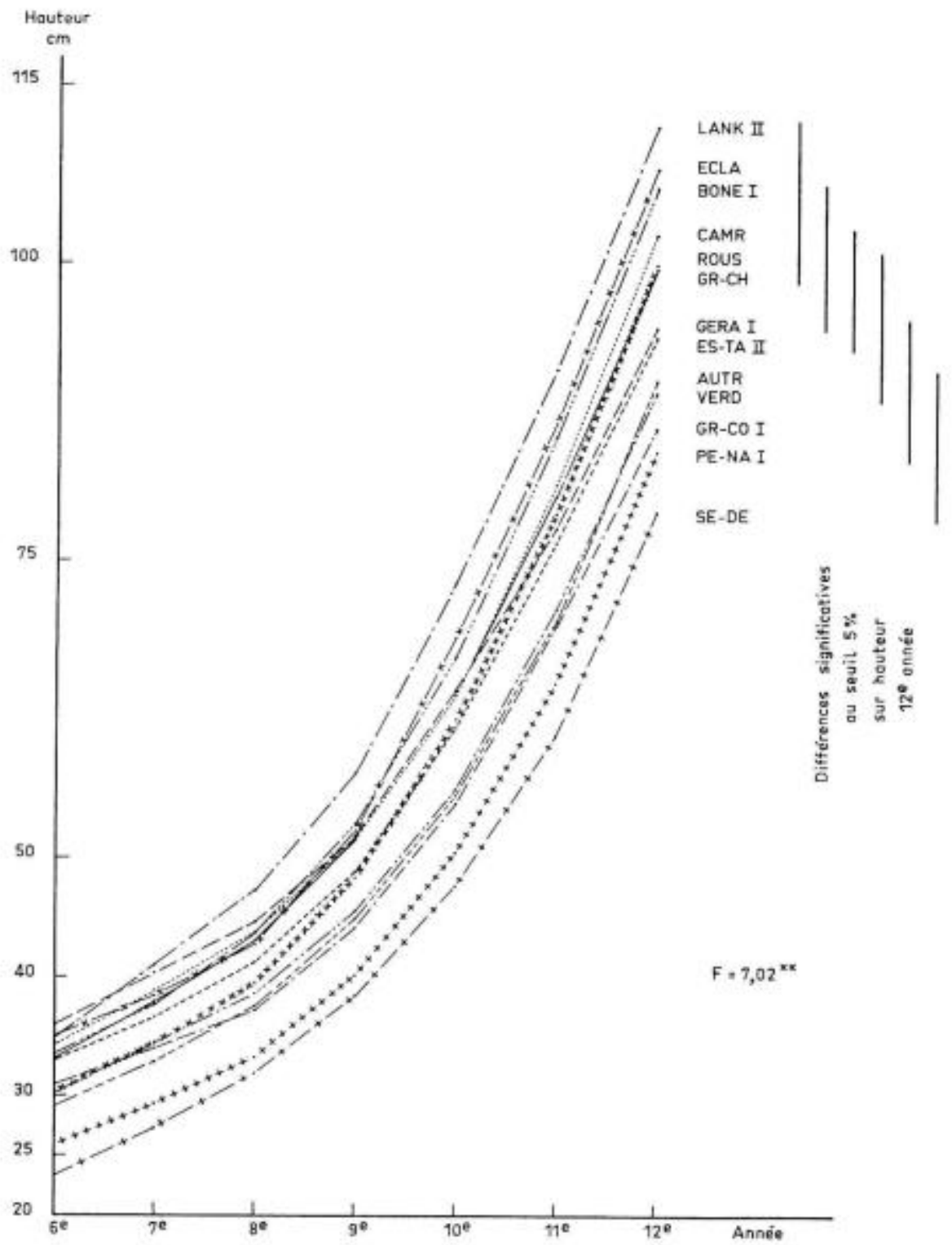

FIG. 6. - Guéry: évolution des hauteurs totoles moyennes des diverses provenances 


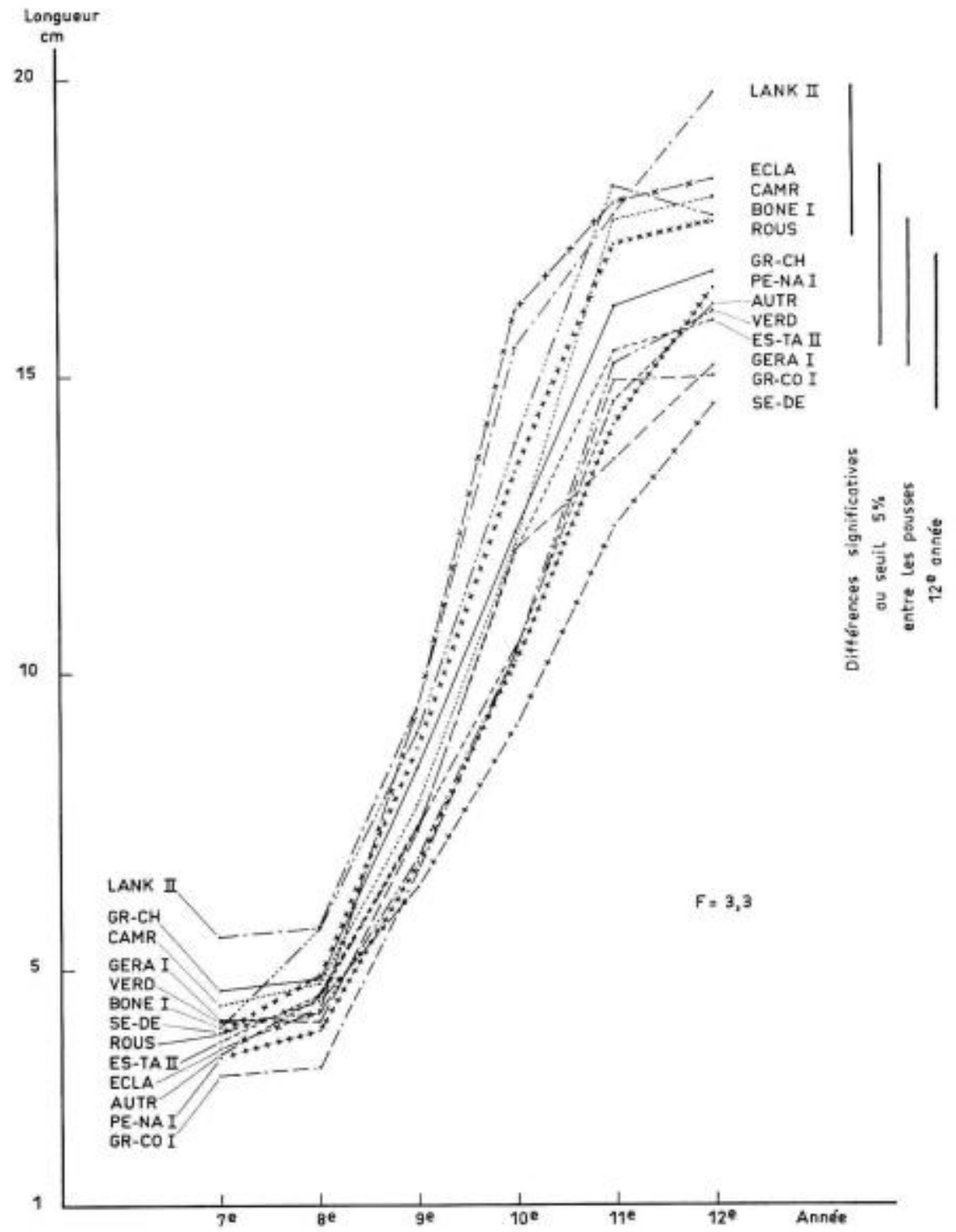

F16. 7. - Guéry : évolution des pousses annuelles moyennes des diverses provenances 
Le classement de hauteur totale à 12 ans s'écarte peu de celui obtenu en pépinière à une exception près: la provenance Gérardmer a disparu du lot de tête. L'examen des courbes donnant l'évolution de la longueur moyenne des flèches en fonction du temps confirme ce point de vue.

Les notations de débourrement permettent de classer les provenances dans un ordre très voisin de celui établi au cours de la quatrième année en pépinière.

\section{III. - COMPARAISON ENTRE STATIONS - INTERACTION}

\section{1. - Niveau de fertilité des trois stations}

Le niveau de fertilité (au sens agronomique du mot) est très différent dans les trois stations. Si nous nous référons aux seules provenances communes aux trois plantations, les moyennes de hauteur à 12 ans se répartissent comme suit (on peut supposer qu'en 1969, la crise de transplantation est terminée dans les trois stations) :

\begin{tabular}{|c|c|c|c|}
\hline Provenances & Grandsagnes & Epinal & Guéry \\
\hline GERA I & 165.1 & 246,8 & 94,5 \\
\hline LANK $\| \ldots \ldots \ldots \ldots . . . . . . . .$. & 178,2 & 240.5 & 111,5 \\
\hline ES-TA $11 \ldots \ldots \ldots \ldots \ldots$ & 162,0 & 216,5 & 93,7 \\
\hline GR-CH $\ldots \ldots \ldots \ldots \ldots \ldots$ & 163,3 & 205,7 & 99,5 \\
\hline VERD $\ldots \ldots \ldots \ldots$ & 174,6 & 199,8 & 89,9 \\
\hline GR-CO 1 . & 164,6 & 210,2 & 86,0 \\
\hline Moyenne générale.......... & 168,1 & 220,0 & 96,1 \\
\hline
\end{tabular}

Si l'on attribue la valeur 100 à la station d'Epinal, on obtient approximativement le niveau 76 pour Grandsagnes et 44 pour le Guéry. Il est à noter que ces écarts s'expliquent facilement par des différences d'altitude, donc de longueur de saison de végétation.

\section{2. - Interaction}

L'intérêt essentiel d'une expérimentation multi-stationnelle consiste à rechercher l'existence d'une éventuelle interaction génotype/milieu qui se traduirait par des changements de classement des provenances.

Nous remarquons, au préalable, qu'une analyse classique susceptible de faire apparaître une source de variation interaction globale significative suppose une certaine identité entre les divers dispositifs. Dans le cas particulier, l'adoption de dispositifs en bloes incomplets équilibrés, assez différents selon les stations, interdit pratiquement cette voie de travail. 
C'est pourquoi nous avons adopté la formule consistant à étudier les liaisons entre moyennes des mêmes caractères des trois dispositifs.

\subsection{Mesures de vigueur.}

Les coefficients de corrélation entre les hauteurs totales (moyennes de provènances), huit ans après plantation (P 1969), des trois dispositifs pris deux à deux ont les valeurs suivantes (calculées à partir des provenances communes) :

Grandsagnes - Guéry ........ 0.44 NS (nombre de degrés de liberté : 5)

Grandsagnes - Epinal ........ 0,08 NS (nombre de degrés de liberté : 5)

Epinal - Guéry ............ $0.81^{\text {** }} \quad$ (nombre de degrés de liberté : 9)

On pourrait en conclure qu'il n'existe pas d'interaction significative globale entre Epinal et Guéry, bien qu'il s’agisse de stations écologiquement très différentes. Néanmoins, la loi de régression (figure 8) montre, par exemp'e, que Gérardmer ne se comporte plus au Guéry comme une provenance à croissance rapide.

On discerne, au contraire, une certaine interaction entre Grandsagnes et les deux autres stations, celle-ci étant plus accusée entre Epinal et Grandsagnes qu'entre Guéry et Grandsagnes. Ce résultat est donné avec une certaine prudence compte tenu du faible nombre de degrés de liberté en cause, mais il faut souligner le déclassement, à Grandsagnes et au Guéry, de la provenance Gérardmer.

Nous pensons done que le phénomène interaction génotype/station, ne peut pas être négligé en matière de provenances d'épicéa et dans le cadre du territoire français. compte tenu de la diversité des stations où l'on utilise l'épicéa en reboisement.

Mais, compte tenu des objectifs de ce type d'expérimentation, il nous semble plus logique d'aborder ce phénomène au niveau de chaque provenance plutôt que d'une manière globale et abstraite.

Les provenances non soumises à interaction seraient par définition celles qui, dans un échantillon donné de populations, conservent pour le caractère étudié un rang à peu près constant. Il s'agit de provenances plastiques (au sens forestier du terme). Les provenances qui échappent à tout effet d'interaction, et qui, en même temps. occupent un bon classement présentent un intérêt particulier. Ce sont des populations \& + méritant une large diffusion dans les reboisements. Dans le cas particulier, la provenance Lankowitz semble pouvoir être rangée dans cette catégorie pour le caractère vigueur. Il en serait de même des sources Eclache et Bonnétage bien qu'elles ne soient représentées que dans deux stations. A l'opposé, la source Gérardmer aurait des exigences plus strictes et ne conviendrait pas pour les stations exposées à des conditions difficiles.

Dans l'expression interaction génotype/milieu, nous avons jusqu'à présent raisonné en terme de génotype global de population. II semble intéressant également d'aborder le problème au niveau des génotypes individuels. En d'autres termes, y a-t-il des changements de classement de sujets au sein des populations?

Les investigations ont été concentrées sur la provenance Gérardmer à Grandsagnes qui, nous l'avons vu, est soumise globalement à l'effet d'interaction.

Pour ce faire, nous admettons que, dans chaque provenance, les classements de hauteur des plants, à la fin de lannée 1962 (deux ans après plantation) s'écartaient 
peu du classement à la sortie de la pépinière (nous ne disposons malheureusement pas de données individuelles à ce stade). Les coefficeints de corrélation intrapopulation entre hauteur fin 1968 et hauteur fin 1962 expriment alors la plus ou moins grande stabilité des classements individuels.

A Grandsagnes, pour toutes les provenances, ces coefficients sont voisins de 1, sauf pour Gérardmer $(r=0,75)$, ce qui tend à prouver l'existence d'une certaine interaction génotype individuel/milieu dans la population Gérardmer, alors que les autres provenances y échapperaient.

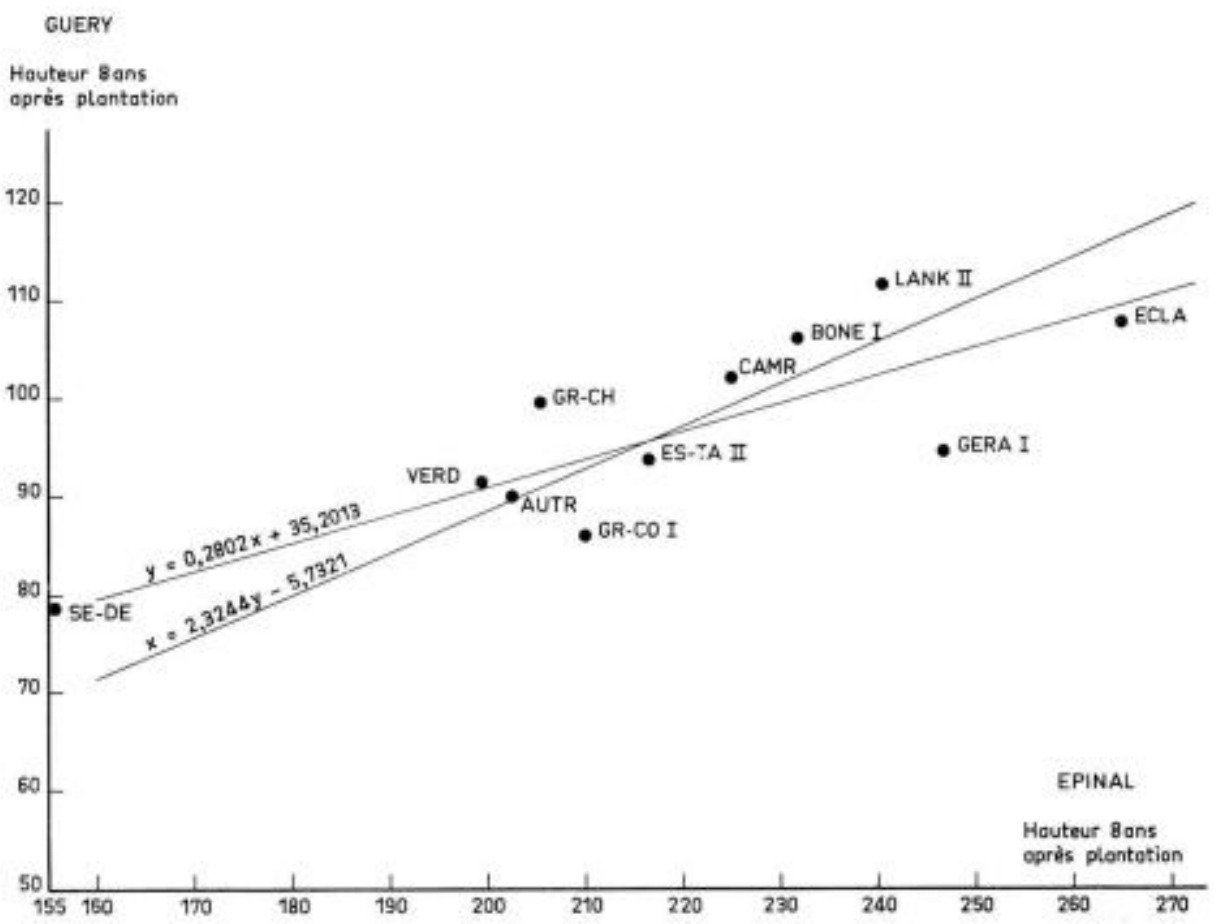

FiG. 8. - Régression entre la hauteur 8 ans après plantation pour Epinal et Guéry

Ceci peut avoir un certain retentissement sur la conception des vergers à graines de familles; on est évidemment tenté d'installer ce type dè verger dans dés régions à climat favorable à la fructification, mais parfois écologiquement éloignées des régions d'utilisation de l'espèce. La sélection effectuée sur le verger, à partir du comportemént des individus et des familles, pourrait aboutir à une impasse dans la mesure où la population de base serait peu plastique donc soumise à interaction.

\subsection{Débourrement.}

On trouvera, ci-dessous, les valeurs des coefficients de corrélation entre les notations moyennes de débourrement de diverses provenances communes aux trois expériences prises deux à deux. 
Epinal-Guéry :

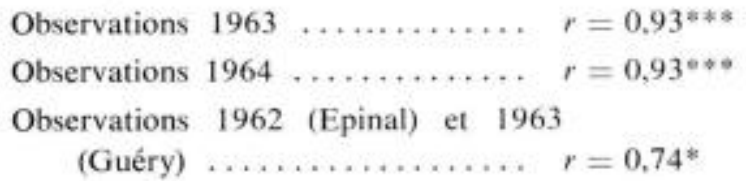

Epinal Grandsagnes:

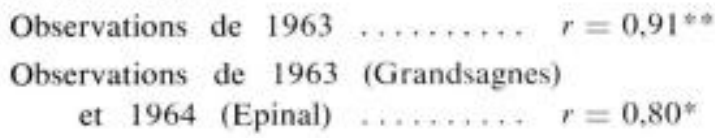

\section{Guéry-Grandsagnes :}

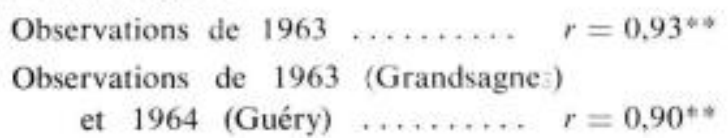

II semble donc que le caractère débourrement végétatif échappe pratiquement à tout effet d'interaction sur station favorable à l'introduction d'épicéa où les besoins en froid de l'espèce sont satisfaits. Il s'agit d'un caractère stable soumis à un contrôle génétique assez rigide.

Le phénomène interaction revêt une grosse importance pratique. La solution idéale consisterait à trouver la population d'élite qui aurait un comportement excellent (vigoureuse, tardive, bonne adaptation) en toutes stations. Dans le cas de l'épicéa. on peut, a priori, douter de pouvoir atteindre cet objectif étant donné que l'espèce est utilisée à divers niveaux d'altitude. En haute altitude (au Guéry, par exemple), ce qui importe c'est avant tout l'aptitude des populations à s'adapter aux conditions rudes du climat. Cette aptitude ne pourra être vérifíe qu'à l'âge adulte (minimum 20 ans après plantation) et de nouveaux changements de classement sont à craindre. dus, par exemple, à des dégâts de cimes sur provenances initialement vigoureusès entrainant des pertes de production etc... C'est la raison pour laquelle il n'est pas possible de conclure définitivement dans ce domaine au stade actuel de l'expériméntation décrite.

\section{IV. - CRISE DE TRANSPLANTATION}

La technique classique de reboisement conduit à procéder à deux transplantations successives, à l'âge de 2 ans (en pépinière) et à l'âge de 4 ans (sur le terrain).

Il n'est pas douteux que cette pratique réduit la précision des résultats obtenus à partir de l'analyse des mesures juvéniles.

L'opération de plantation sur le terrain entraîne inévitablement des lésions en particulier des mutilations et déformations du système racinaire qui augmentent la part des facteurs aléatoires. On peut supposer que les effets de cette crise de transplantation sont temporaires, mais le problème se pose de déterminer à quel moment ils disparais- 
sent ou deviennent négligeables pour éviter d'effectuer des mesures et observations peu efficaces.

La réponse peut varier selon les stations, les provenances, voire les individus.

Les mesures et observations, décrites dans le cas présent, couvrent cette période d'installation dans les trois stations. Nous avons tenté de résoudre le problème posé par trois approches différentes, en partant des mesures de vigueur sensibles aux effets de cette crise :

1) l'évolution dans le temps des coefficients de variation intrapopulation,

2) l'évolution dans le temps des coefficients de variation interpopulation,

3) l'évolution dans le temps des liaisons entre valeurs moyennes de mesures de vigueur des diverses populations.

\section{1. - Evolution dans le temps des coefficients de variation intrapopulation}

Le coefficient de variation (cv) est défini comme étant le rapport de l'écart type d'une variable à sa moyenne. L'écart type qui exprime l'ampleur de la variabilité tend à augmenter avec la grandeur de la moyenne. Aussi, semble-t-il plus logique d'utiliser le paramètre cv pour comparer les variabilités de mesures concernant le même caractère (vigueur) mais échelonnées dans le temps, donc de valeurs moyennes croissantes.

Dans le cas présent, le cv a été calculé à partir de données individuelles, au sein de chaque provenance, pour le caractère longueur de la flèche annuelle et pour les années 1963 à 1968. Les données sont portées sur les figures 9,10 et 11 correspondant aux trois plantations en cause.

Une première remarque concerne l'évolution générale du cv; il tend à augmenter au cours des premières années après plantation, puis à diminuer. Par ailleurs, les écarts de cv ainsi calculés entre provenances s’amortissent progressivement.

Ainsi, à Epinal, le cv intrapopulation se maintient approximativement pour toutes les provenances au niveau 50 jusqu'en 1965, puis retombe à 40 en 1966 et 35 en 1968 .

Au Guéry, l'évolution est beaucoup plus marquée : 60 en 1963, 80 en 1964, 75 en 1965,65 en 1966,35 en 1967 et 1968.

A Grandsagnes, 40 de 1963 à 1965 , puis 30 ultérieurement.

Dans les trois cas, les écarts entre provenances se réduisent considérablement en 1967 et 1968 ,

Il convient de souligner qu'à la fin de la période considérée, dans les trois stations, les cv prennent des valeurs comparables (30 à 35 ).

Comment interpréter ces informations?

Le comportement phénotypique d'un individu $(\mathrm{P})$ est la résultante de deux actions, l'une génotypique $(G)$, l'autre due à l'environnement $(E)$, ce qui s'écrit: $P=G+E$.

Les différents termes de cette équation expriment la déviation par rapport à la moyenne de la population étudiée.

Le phénomène crise de transplantation constitue une source d'augmentation des écarts dus au milieu, donc de $\mathrm{E}$; la crise terminée, $\mathrm{E}$ devrait se stabiliser. L'effet $\mathrm{G}$ 
étant supposé constant, on peut admettre que la fin de la crise de transplantation devrail correspondre avec une stabilité de $\mathrm{P}$ donc du $\mathrm{cv}$. Ce raisonnement suppose l'absence de tout effet concurrence entre plants, ce qui est le cas au stade de développement choisi.

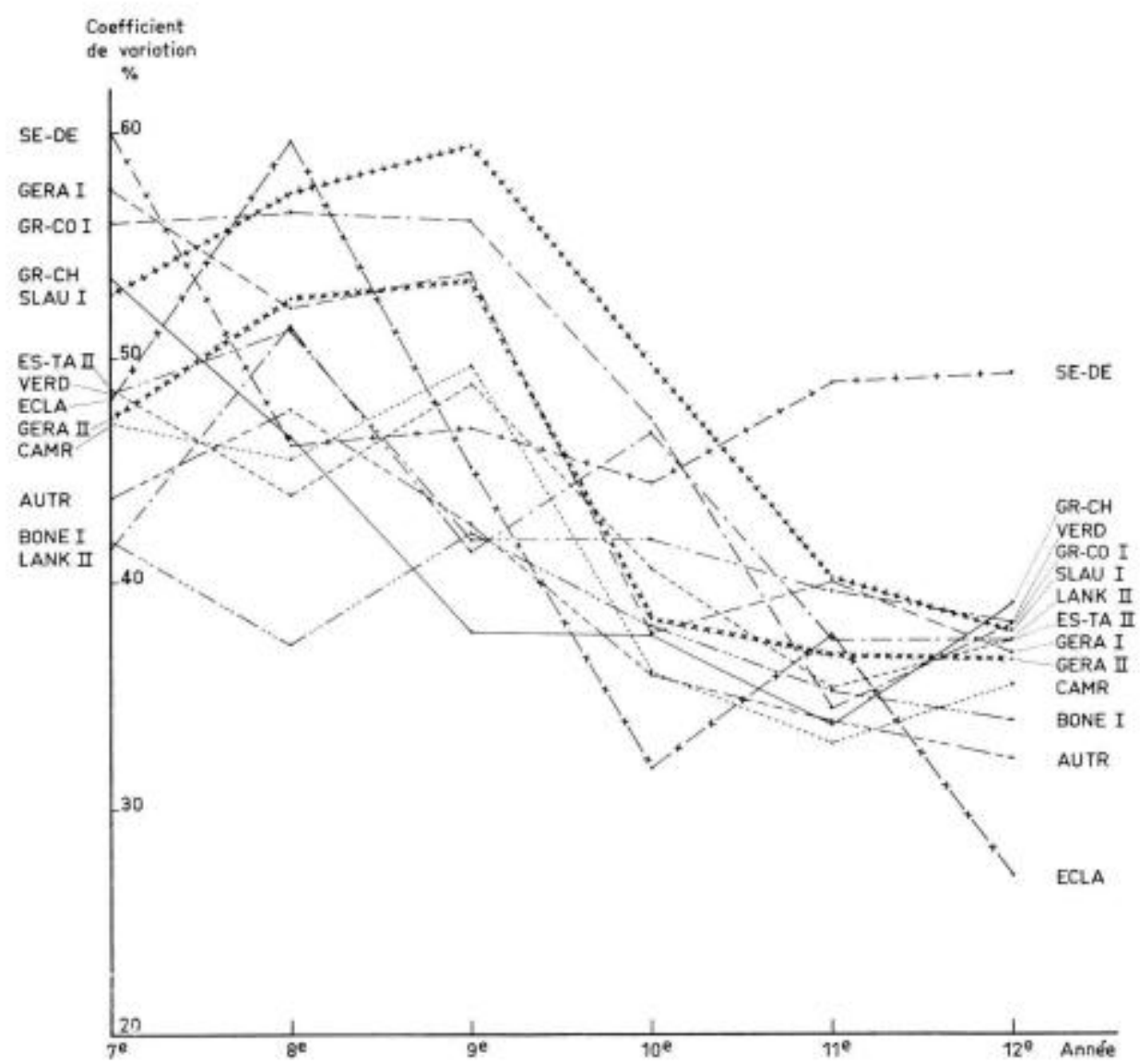

Fia. 9. - Epinal: évolution dans le temps des coefficients de variation intra-provenance de la pousse anmuelle

Dans le cas présent, la crise aurait donc pris fin en 1965 à Grandsagnes, 1965 ou 1966 à Epinal et en 1966 au Guéry. Les valeurs de cv atteignent un palier pour les flèches des années 1966 (Grandsagnes) et 1967 (Guéry). Compte tenu du décalage d'un an entre l'expression de l'élongation et la formation du bourgeon qui la conditionne, nous en concluons que les effets de la crise deviennent négligeables respectivement en 1965 et 1966. Pour Epinal, le phénomène se situe entre ces deux années.

Les comportements des diverses provenances, vus sous cet angle, diffèrent notablement. Ainsi, nous remarquons que pour Bonnétage, représentée au Guéry et à Epi- 


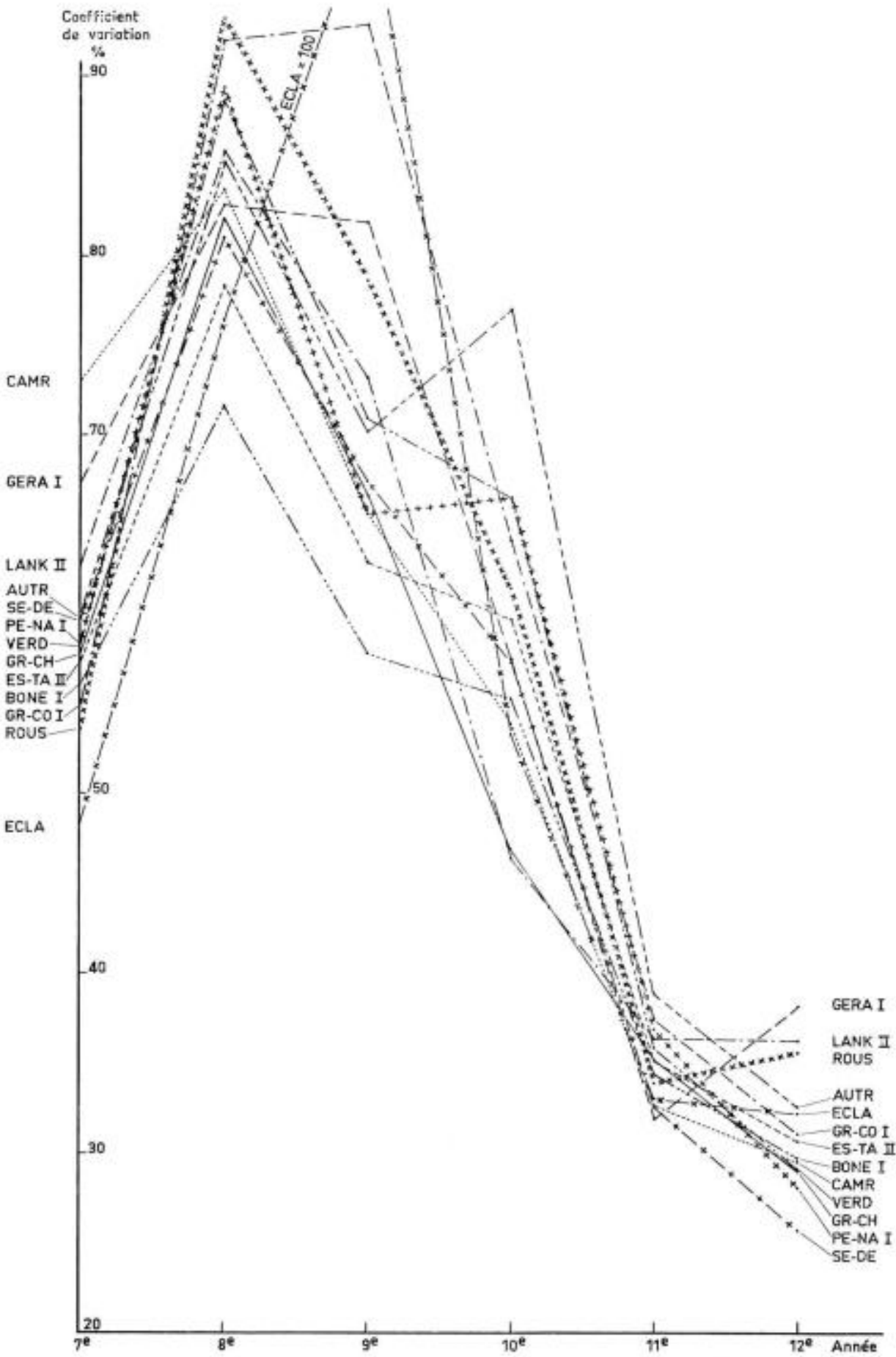

FIG. 10. - Guéry : evolution dans le temps des coefficients de variation intra-provenance, de la pousse annuefle 
nal, la valeur cv passe par un niveau maximal relativement faible et décroît plus rapidement. Cette provenance aurait une meilleure aptitude à la reprise.

Au contraire, Eclache, représentée au Guéry et à Epinal, et Grand-Côte dans les trois stations, éprouveraient des difficultés de transplantation plus marquées.

On peut aussi penser que l'effet des gelées tardives sur les provenances précoces (Eclache) a contribué à augmenter les valeurs de $\mathrm{cv}$ pendant la phase juvénile, alors que ce phénomène n'est pas intervenu pour Bonnétage indemne de tout dommage.

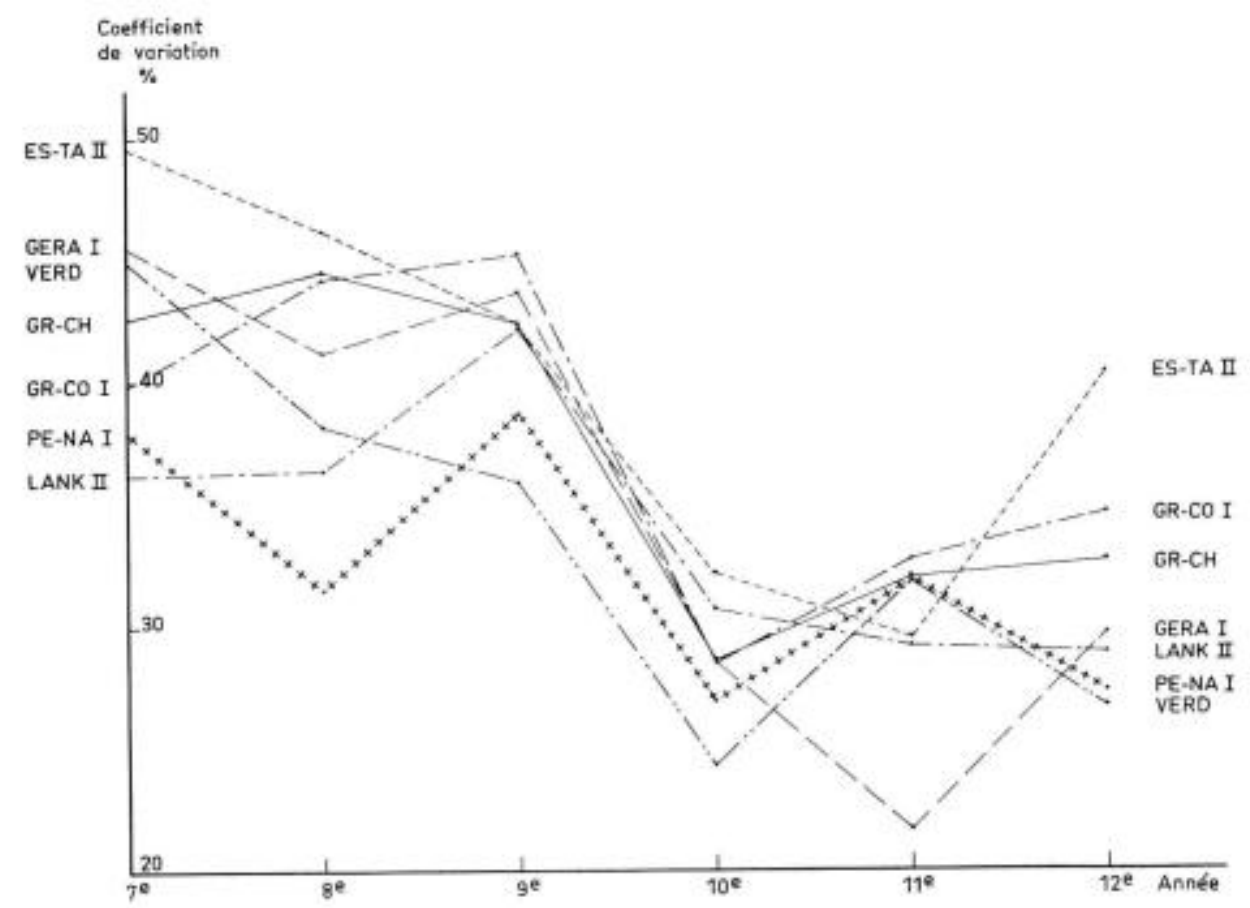

FIG. 11. - Grandsagnes: évolution dans le temps des coefficients de variation intra-provenance de la pousse annuelle

L'explication ne vaut pas pour Grand-Côte, population tardive peu endommagée et dont le cv passe par un maximum de valeur élevée.

\section{2. - Evolution dans le temps des coefficients de variation interpopulation}

II s’agit maintenant d'un coefficient de variation calculé pour chaque mesure de vigueur à partir des valeurs moyennes de toutes les populations (par station).

Ce cv diffère totalement du précédent. Le cv intrapopulation étudié au chapitru 4.1 exprime la variabilité intrapopulation : au contraire, le cv interpopulation fournit une estimation de l'amplitude de la variabilité génétique infraspécifique.

On trouvera sur le tableau 4 les valeurs des cv calculées à partir des mesures de flèche annuelle et de hauteur totale, pour chaque année, dans les trois stations. 


\section{TABLEAU 4}

Valeurs des coefficients de variation (cv) interpopuiation

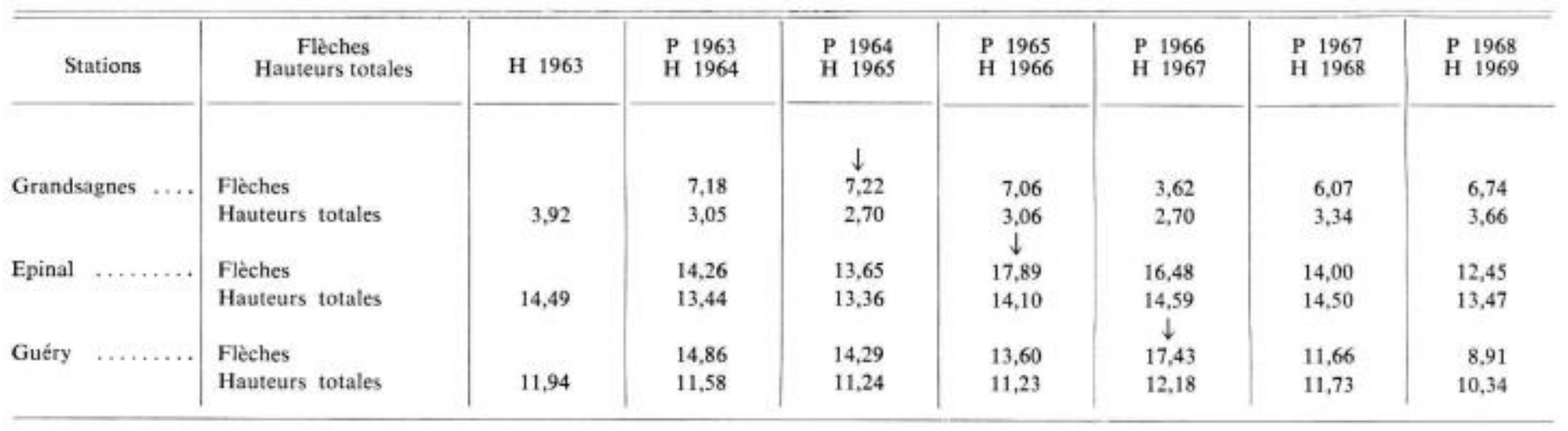

\. Localise la fin présuméz de la crise de transplantation.

H 1963 = Hauteur totale fin 1962 ,

P 1963 = Pousse de lannće 1963. 
Au cours des années suivant la plantation, ces cv suivent l'évolution générale sulvante : ils commencent par diminuer, puis leur valeur augmente, passe par un maximum et tend à diminuer de nouveau. Il est vraisemblable que la crise de transplantation, qui provoque des troubles de croissance incontrôlés, se traduit par une réduction de ce cv: ceci explique la première phase au cours de laquelle la variabilité infraspécifique est partiellement masquée par des causes externes (crise). Le stade ultérieur (augmentation du cv) coïnciderait avec la fin présumée des effets de crise de transplantation qui se situerait en 1964 pour Grandsagnes et Epinal, et en 1966 pour le Guéry, ceci confirmant à peu près les résultats du chapitre précédent.

La diminution ultérieure du cv pourrait susciter quelques inquiétudes. Faut-il conclure que la variabilité infraspécifique constatée au stade juvénile tend à décroître avec le temps? Ce phénomène, déjà constaté par NANSON 11 , semble assez général dans les études de variabilité infraspécifique basées sur le caractère hauteur. Une analyse à partir de la variable volume conduirait à des conclusions inverses. Dans le cas présent, une explication vient à l'esprit : le $\mathrm{cv}$ interpopulation concernant les mesures de hauteur ne peut que diminuer dans le temps, dans la mesure où l'échantillon de populations étudié comporte des provenances à élongation initiale lente mais plus rapide dans un stade ultérieur ; or, nous avons déjà constaté que certaines provenances appartiennent ici à cette catégorie (Saint-Etienne-en-Dévoluy et Saint-Laurent).

\section{3. - Evolution dans le temps des liaisons entre valeurs moyennes des mesures de vigueur des diverses populations}

Ces liaisons sont exprimées par les coefficients de corrélation entre diverses valeurs moyennes de mesures de vigueur échelonnées dans le temps. Les valeurs de ces coefficients figurent sur le tableau ci-dessous.

\begin{tabular}{|c|c|c|c|c|c|c|c|}
\hline & & 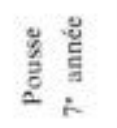 & 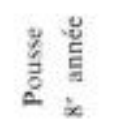 & 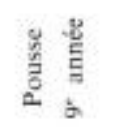 & 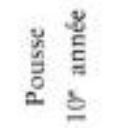 & 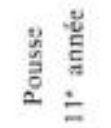 & 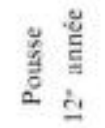 \\
\hline $\begin{array}{l}\text { Grandsagnes : } \\
(5 \text { degrés de } \\
\text { liberté) } \ldots . . . .\end{array}$ & $\begin{array}{c}\text { Hauteur aे } \\
12 \text { ans (terrain) }\end{array}$ & $0,19 \mathrm{NS}$ & $0,67 \mathrm{NS}$ & $0,75^{*}$ & $0,85^{*}$ & $0,83^{*}$ & $0.80^{\bar{\psi}}$ \\
\hline $\begin{array}{l}\text { Epinal: } \\
\text { (11 degrés de } \\
\text { liberté) } . . . . .\end{array}$ & $\begin{array}{l}\text { Hauteur } \\
\text { â } 12 \text { ans }\end{array}$ & $0,77^{* 4}$ & $0,94^{\text {eat }}$ & $0,97^{* * * *}$ & $0,96^{\circ \cdots 4}$ & $0,96^{=5 *}$ & $0,90^{\neq * 2}$ \\
\hline $\begin{array}{l}\text { Guéry: } \\
\text { (11 degrés de } \\
\text { liberté) } \ldots . . .\end{array}$ & $\begin{array}{l}\text { Hauteur } \\
\text { à } 12 \text { ans }\end{array}$ & $0,59 *$ & 0,60 & $0,95 * \approx$ & $0,96 \%$ & $0,90^{* 3 *}$ & $0,89 * * *$ \\
\hline
\end{tabular}

(1) Contribution à l'étude de la valeur des tests précoces. II. Expérience internationale sur l'origine des graines de Pin sylvestre (1906). Trav. Sin. Rech, Groenendaal, N" E 2, 42, P. 1967. 
Nous considérons que, dans chaque station, le classement de vigueur obtenu à partir des mesures de hauteur totale à 12 ans ( 8 ans après plantation) n'est pratiquement plus perturbé par les effets de la crise de transplantation. Les coefficients de corrélation entre cette mesure et celles des flèches des années suivant la plantation augmentent puis atteignent un palier correspondant à une valeur généralement élevée. On peut admettre, en gros, que la fin de la crise coïncide avec le début de ce palier.

Les effets de cette crise deviendraient donc négligeables en 1966 à Grandsagnes, en 1964 à Epinal et en 1965 au Guéry.

Ces résultats diffèrent de ceux obtenus par les deux premières méthodes en particulier en ce qui concerne Grandsagnes (longueur de la crise plus longue d'après la troisième méthode). L'explication de cette anomalie est facile : nous avons décelé dans cette station une certaine interaction génotype/milieu. Ceci signifie que le classement des populations à l'issue de l'élevage en pépinière d'Amance tend à se modifier à Grandsagnes; mais le phénomène intervient progressivement, d'où la nécessité d'un certain délai avant d'obtenir le classement définitif.

Le phénomène crise de transplantation affecte donc largement l'expression de la vigueur. En est-il de même pour le débourrement végétatif? $\mathrm{Ne}$ disposant pas d'un nombre aussi important de mesures échelonnées dans le temps, il n'est pas possible d'aborder le problème selon le même processus.

Nous ferons l'hypothèse que le classement obtenu en pépinière au début de la 4* année (printemps 1960) s'écarte peu du classement génétique réel, pour le comparer à ceux résultant des observations sur le terrain dans les trois stations. Les liaisons sont exprimées ci-dessous par les coefficients de corrélation correspondants.

\begin{tabular}{|c|c|c|c|c|}
\hline & $\begin{array}{l}\text { Débourrement } \\
6^{\circ} \text { année }\end{array}$ & $\begin{array}{l}\text { Débourrement } \\
7^{*} \text { année }\end{array}$ & $\begin{array}{l}\text { Débourrement } \\
8^{\circ} \text { année }\end{array}$ & \\
\hline $\begin{array}{l}\text { Débourrement } 4^{*} \text { an- } \\
\text { née } \ldots . . \ldots \ldots \ldots \ldots . \ldots\end{array}$ & $0,78=7$ & 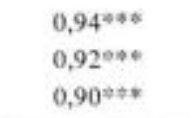 & $\begin{array}{l}0,92^{\circ, 00} \\
0,72^{\text {pop }}\end{array}$ & $\begin{array}{c}\text { Grandsagnes } \\
\text { Epinal } \\
\text { Guéry }\end{array}$ \\
\hline
\end{tabular}

Il semble donc que, contrairement à la vigueur, ce caractère soit peu perturbé par la crise, ce qui confirme une fois de plus qu'il est sous contrôle génétique rigide.

En conclusion, nous constatons que pour l'épicéa, les mesures de vigueur effectuées sur les plantations comparatives classiques de provenances au cours des quatre et cinq premières années suivant l'installation fournissent des informations peu précises. Ce résultat suggère deux réflexions:

la première concerne les programmes de mesures qu'il conviendrait d'alléger en se limitant aux seules mesures de date de débourrement au cours des premières années :

la deuxième a une portée plus générale. Les techniques de reboisement habituellement adoptées en France, pour l'épicéa (grands plants, traumatismes importants des racines), comportent le risque d'une longue crise de transplantation; celle-ci, nous 
l'avons vu, tend à réduire notablement les effets positifs d'une sélection génétique pour la vigueur pendant la période juvénile. Ceci est d'autant plus regrettable que l'on s'accorde à reconnaitre l'intérêt des variétés à croissance initiale rapide (économie sur les dégagements).

\section{V. - PREVISIONS DE COMPORTEMENT - TESTS PRECOCES}

Une préoccupation constante des responsables des programmes d'amélioration est de rechercher l'existence de liaisons entre caractères juvéniles et caractères de l'adulte.

Dans le cas présent, nous avons mis en parallèle les informations obtenues en pépinière et celles recueillies sur les plantations.

Mesures de hauleur $t$

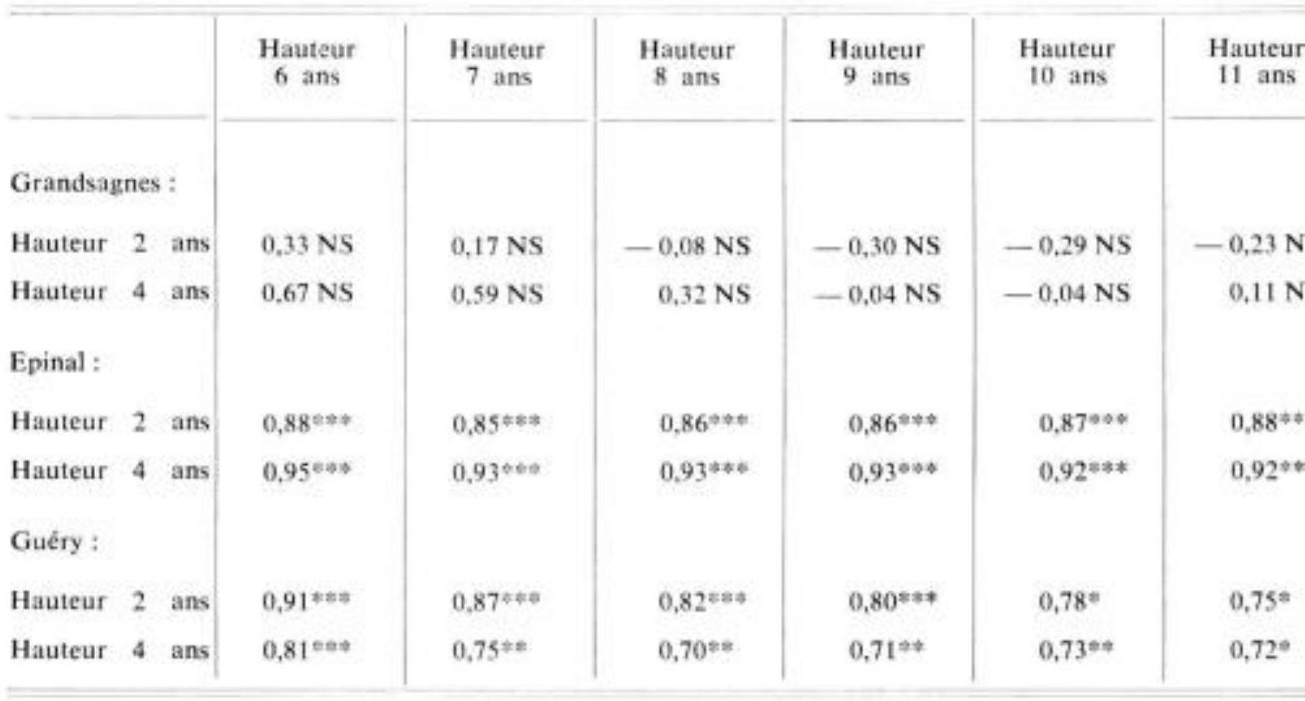

\section{1. - Débourrement végétatif}

Nous avons souligné dans le chapitre précédent l'intensité de la liaison entre les classements obtenus en pépinière et sur le terrain. Les notations ayant été effectuées sur le terrain au cours des quatre années suivant la plantation, donc pendant la période où la date de débourrement revêt une importance pratique (arbres de petite taille, vulnérables aux gelées tardives qui affectent le plus souvent les couches d'air basses). Les liaisons sont telles que l'on peut affirmer que pour le caractère en cause, les trois plantations comparatives n'apportent aucune information nouvelle par rapport à celles fournies en pépinière; ceci malgré la diversité écologique des stations et quelle que soit la gravité de la crise de transplantation. 
En conclusion, il semble que des essais à très court terme. non répétés dans l'espace, sont susceptibles de fournir des classements de populations suffisamment précis,

\section{2. - Vigueur}

Les possibilités de prévisions précoces de vigueur sont moins évidentes. C'est ce qui ressort de l'examen des coefficients de corrélation entre les mesures de hauteur totale et de pousses annuelles. effectuées dans les trois stations de 1963 à 1969 (tableau 5).

Les informations obtenues en pépinière sont démenties à Grandsagnes. II ne faut pas s'en étonner compte tenu du phénomène interaction particulièrement accusé dans

pousses annuelles

\begin{tabular}{|c|c|c|c|c|c|c|}
\hline $\begin{array}{l}\text { Hauteur } \\
12 \text { ans }\end{array}$ & $\begin{array}{l}\text { Pousses } \\
77 \text { année }\end{array}$ & $\begin{array}{l}\text { Pousse } \\
8^{\circ} \text { année }\end{array}$ & $\begin{array}{l}\text { Pousse } \\
9 \text {. annéc }\end{array}$ & $\begin{array}{l}\text { Potisse } \\
\text { for annee }\end{array}$ & $\begin{array}{l}\text { Pousse } \\
\text { II annét }\end{array}$ & $\begin{array}{l}\text { Pousse } \\
12 \text { année }\end{array}$ \\
\hline $0,35 \mathrm{NS}$ & $-0,51 \mathrm{NS}$ & $-0.58 \mathrm{NS}$ & $-0,53 \mathrm{NS}$ & $-0.18 \mathrm{NS}$ & $-0,10 \mathrm{NS}$ & $-0.55 \mathrm{NS}$ \\
\hline $0,09 \mathrm{NS}$ & $-0.40 \mathrm{NS}$ & $-0,50 \mathrm{NS}$ & $-0,60=$ & $-0.31 \mathrm{NS}$ & $-0.24 \mathrm{NS}$ & $-0,50 \mathrm{NS}$ \\
\hline $0,85^{\text {tod }}$ & $0,52 \mathrm{NS}$ & $0.89=54$ & $0.81^{0.040}$ & $0,88^{\text {하나에 }}$ & $0,910.60$ & 0,76 ese \\
\hline 0.92 & $0.59=$ & 0.92000 & $0,88^{=0}=$ & $0,86^{\text {max }}$ & $0.88^{000}=$ & $0,87 \neq=8$ \\
\hline $0,69=8$ & $0,23 \mathrm{NS}$ & $0,18 \mathrm{NS}$ & $0.62=$ & $0.70 \Rightarrow$ & $0,56=$ & $0,39 \mathrm{NS}$ \\
\hline $0,67^{\circ e}$ & $0.06 \mathrm{NS}$ & $0.09 \mathrm{NS}$ & $0,62=$ & 0,74 ov & $0.59=$ & $0,40 \mathrm{~N}^{r}$ \\
\hline
\end{tabular}

cette station. Les liaisons entre données juvéniles de pépinière et pousses annuelles sur le terrain sont négatives. Celles concernant les hauteurs totales diminuent progressivement. ce qui confirme la s rémanence * des classements en pépinière.

En ce qui concerne le Guéry, ces corrélations suivent une évolution intéressante : elles sont d'abord faibles (crise de transplantation), puis elles augmentent et tendent ensuite à décroître. ce qui peut indiquer qu'un effet interaction commence à s'exprimer.

A Epinal, en labsence de toute interaction et avec une crise de transplantation moins marquée. on peut considérer qu'il n'existe aucune divergence entre les informations fournies par la plantation comparative et celles venant de la pépinière. Ceci est logique, puisque des trois stations en cause. Epinal est celle dont les conditions écolo- 
giques sont à la fois les plus favorables et les plus proches de celles de la pépinière d'Amance.

En conclusion, des prévisions précoces de comportement de vigueur de populations d'épicéa semblent possibles avec une précision très satisfaisante en l'absence d'interaction génotype/milieu lorsque les stades juvénile et ultérieur se situent dans des milieux différents.

\section{V1. - MODIFICATIONS DE LA METHODOLOGIE DES PLANTATIONS COMPARATIVES}

La méthode classique des plantations comparatives adoptée pour l'étude des populations de résineux consistant à concentrer les élevages dans une seule pépinière, puis à répartir les plants entre diverses expérimentations à moyen ou long terme $(1 / 3$ à $1 / 2$ de la révolution), comporte, nous le constatons, un certain nombre d'inconvénients,

Les crises de transplantation (deux transferts des plants) et l'existence d'une éventuelle interaction contribuent à augmenter les délais nécessaires avant l'établissement de bilans objectifs, c'est-à-dire à réduire l'efficacité de l'investissement recherché.

Par ailleurs, cette procédure se traduit par l'immobilisation de surfaces importantes pendant de longs délais, au point d’être pratiquement inutilisable lorsque le nombre de populations à étudier est élevé.

Compte tenu des conclusions des chapitres précédents, nous proposons une méthodologie différente, qui correspond à un compromis entre les exigences techniques et les possibilités matérielles, et qui semble recueillir, sous des formes voisines, la faveur de certains chercheurs étrangers. Cette nouvelle méthode comporterait deux étapes successives.

\section{Première étape}

Celle-ci consisterait à créer des expérimentations présentant les caractéristiques suivantes :

- Court terme: 7 à 20 ans selon le rythme de croissance juvénile de l'espèce étudiée et la fertilité de la station. Le court terme autorise l'utilisation d'écartements faibles entre plants $(0,50$ à $1 \mathrm{~m})$ et permet une économie de surface appréciable.

A titre d'indication, un dispositif comportant 6 répétitions avec des parcelles unitaires de 30 plants espacés de $1 \mathrm{~m}$ permet de comparer 55 populations à l'hectare, au lieu de 6 avec la méthode actuelle.

- Installation sur une station aussi homogène que possible après une préparation intensive en vue de réduire au maximum les effets fertilité et utilisation, dans certains cas, de techniques particulières; exemple : système de protection contre les gelées tardives pour les études sur les sapins pectinés.

- Répétition de l'expérimentation sur plusieurs stations; le nombre de ces répétitions dépendrait de l'amplitude de la variabilité écologique de la zone d'introduction de l'espèce, mais il conviendrait de ne retenir que des stations suffisamment différentes 
pour justifier l'existence d'une éventuelle interaction génotype/milieu. Aussi, pour l'épicéa, nous proposons au maximum quatre implantations:

Basse altitude atlantique (sud-ouest).

Basse altitude nord-est, semi-continentale.

Altitude Massif Central.

Moyenne altitude sud-sud-ouest du Massif Central.

Dans de nombreux cas, une implantation donnée pourrait convenir à plusieurs essences, d'où la possibilité de concentration des dispositifs.

- Utilisation directe de jeunes semis: les deux transplantations successives seraient éliminées en utilisant de jeunes plantules (1 à 3 mois) élevées éventuellement en godet de carton, ce qui présente deux avantages :

réduction sérieuse de l'effet crise de transplantation,

possibilité de déceler très rapidement l'effet interaction.

Il s'agit d'une technique difficilement transposable dans la pratique des reboiseurs, mais dont l'intérêt consiste à découvrir le plus rapidement possible la variabilité génétique.

Il est permis de l'adopter compte tenu des précautions prises pour la préparation du sol et éventuellement la protection des plants.

\section{Deuxième étape}

On reprendrait, dans le cadre d'essais à moyen ou long terme, les populations les mieux placées dans les essais de la première étape. Le faible nombre de populations retenues permettrait d'adopter des dimensions de parcelles unitaires compatibles avec les exigences de calculs de production. Ils permettraient aussi de juger des caractères mesurables seulement chez l'adulte (exemple : forme, épaisseur de l'écorce).

Ce schéma est volontairement simplifié. Des formules intermédiaires sont possibles comme par exemple la prolongation de certains dispositifs à court terme, par des éclaircies de populations.

\section{CONCLUSION}

L'analyse de cette expérimentation sur les provenances d'épicéa nous permet d'informer les reboiseurs sur les mérites respectifs des populations étudiées, en fonction des critères retenus.

Si l'objectif est de découvrir des provenances $<+\$$ se classant favorablement en toutes stations pour tous les caractères, il faut bien reconnaître qu'aucune de celles qui sont représentées ne peut recevoir ce label. La provenance Lankowitz, vigouréuse dans les trois stations, se révèle malheureusement très précoce.

Malgré les imperfections de la méthode suivie, nous décelons l'existence d'une certaine interaction génotype/milieu, affectant plus particulièrement certaines populations (Gérardmer). 
Cette analyse nous suggère enfin certaines réflexions sur la méthodologie adoptée systématiquement pour les comparaisons de populations de conifères et nous conduit à proposer des modifications importantes susceptibles d'améliorer l'efficacité des travaux. mais entrainant une remise en catuse de la stratégie des programmes.

Recu pour publication en decembre 1969.

\author{
SUMMARY
}

ANALYSIS OF A MULTISTATIONAL EXPERINENT ON SEVERAI SPRUCE-PROVENANCES

(Picea abies kakst)

This paper accounts fot an experiment on a mainly french spruce provenance test. initiated in 1957 (sowing). The seedlings were grown for 4 years in the Amance nursery (near Nancy) and distributed among for plantations located on three sites whose ecological conditions are quite differenı (Epinal, Guéry. Grandsagnes).

The analysis done in each location shows that none of the studied provenances have a good behaviour for both characters: vigor and late flushing. The three more vigorous populations, Gerardmer (Vosges). Eclache (artificial) and Lankowitz (Austria) flush early.

When comparing the results from the three sites, it is possible to detect a certain genotype/site interaction effect. This is specially strong between the two remotest sites, Epinal and Grandsagnes, for vigor, The Gerardmer population, vigorous at low altitude. is no more fast growing under severe ecological conditions. On the contrary, the ranking of populations for flushing is quite stable in all sites.

It has been possible to calculate length and importance of the transplantation crisis. by analysing its effect on intra and interpopulation variability of vigor and on correlation between population vigor measurements done at different ages.

This study allowes to do a critical study of the experimental method adopied in France for conifer population lests and to suggest a different procedure. A first step will consist in short term tests, replicated in different sites, using very young seedlings planted on homogeneous and intensively cultivated land. This in order to reduce the transplantation crisis effect and to detect as rapidly as possible any interaction effect and improve the efficiency of research work (more precise results obtained more rapidly). The populations sorted out at the end of the first type of experiments, may be used in long term experiments (second step) in order to obtain informations on yield or any other adult characters.

\title{
ZUSAMMENFASSUNG
}

VERGIEICH DES AUFVERSCHIEDENEN STANDORTEN DURCHGEFLI:ARTEN FICHTEN-PROVENIENZ. VESUCHES (Picea abies KARST)

Die vorliegende Arbeit bringt die Eryebnisse eines Fichten-Provenienz versuches mit vorwiebend französischen Herkünften. welcher 1957 (Sämlinge) begonnen wurd:. Die Pflanzen wurden im Pflanzgarten von Amance in der Nähe von Nancy aufgezogen und vierjïhrig. in Vergleichespflanzungen auf drei, ökologisch sehr verschiedenen Standorten: ausgepflanzt (Epinal, Guéry, Grandsagnes).

Die Untersuchungen auf den drei Standorten erbrachten keine zufriedenstellende Reihung der Herkünfte bezuiglich der beiden Selektionsmerkmale: "Wuchskraft ? (vigueur) und s. Spättreibens. Die drei wuchskräftigsten Herküfte: Gerardmer (Vogesen). Eclache (künstliche Herkunft) und Lankowitz (österreich) sind gleichzeitig Frühtreiber.

Der Vergleich der auf den drei Standorten erzielten Ergebnisse lässt auf eine gewiss: Interaktion Genotyp-Standort schliessen. Diese Interahtion ist vor allem bei dem Merkmal 
* Wuchskraft \& zwischen den beiden am weitesten voneinander entfernten Standorten Epinal und Grandsagne sehr deutlich. Dies zeigt sich insbesondere bei der Herkunft Gerardmer, welche in geringer Seehöhe sich als sehr wuchskräftig erweist, unter ungünstigeren Bedingungen jedoch stärker zurückbleibt. Die Reihung nach dem Merkmal \& Austrieb s scheint im Gegenteil räumlich sehr stabil.

Im weiteren konnte die Dauer und Grösse des Auspflanzschockes ermittelt werden und 2war durch die Abschätzung sainer Wirkung auf die Variabilitït des Merkmales \& Wuchskraft"» innerhalb und zwischen den Herkünften sowie auf die Intensität der Beziehungen zwischen den periudischen Wuchskraftmessungen an den Herkünften.

Schlicsslich erlaubt die Bilanz dieses Versuches eine kritische Analyse der in Frankreich angewandten Versuchsmethodik an Nadelhölzern und führt zu einem neuen Versuchsablauf. In einem ersten Stadium wären kurzfristige räumlich wiederholte Versuche anzulegen. wobei auf homogenem Boden intensiv aufgzzogene Pflanzen verwendet werden sollten. Man hofft damit den Auspflanzschock zu vernindern und sehr rasch eine eventuelle Interaktion feststellen zu können. Das frühzeitige Vorliegen genatter Ergbnissz führt damit zu einer Verbzsserung der Wirksamheit der Forschungsarbeit.

Im folgenden Stadium werden einige wenige, in den kurzfristigen Versuchen ansgewählte Herkünfte, in ein langfristiges Programm aufgenommen, welches vor allem die Gesamtwuchsleistung sowie Merkmale des erwachsenen Baumes unteisuchen soll. 